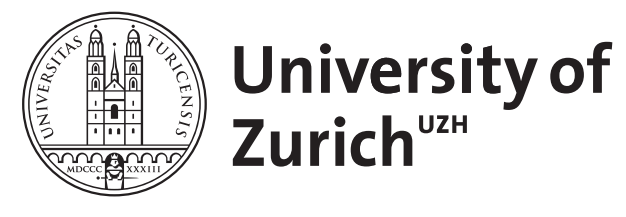

\title{
Lattice diffusion of a single molecule in solution
}

\author{
Ruggeri, Francesca ; Krishnan, Madhavi
}

\begin{abstract}
The ability to trap a single molecule in an electrostatic potential well in solution has opened up new possibilities for the use of molecular electrical charge to study macromolecular conformation and dynamics at the level of the single entity. Here we study the diffusion of a single macromolecule in a two-dimensional lattice of electrostatic traps in solution. We report the ability to measure both the size and effective electrical charge of a macromolecule by observing single-molecule transport trajectories, typically a few seconds in length, using fluorescence microscopy. While, as shown previously, the time spent by the molecule in a trap is a strong function of its effective charge, we demonstrate here that the average travel time between traps in the landscape yields its hydrodynamic radius. Tailoring the pitch of the lattice thus yields two different experimentally measurable time scales that together uniquely determine both the size and charge of the molecule. Since no information is required on the location of the molecule between consecutive departure and arrival events at lattice sites, the technique is ideally suited to measurements on weakly emitting entities such as single molecules.
\end{abstract}

DOI: https://doi.org/10.1103/PhysRevE.96.062406

Posted at the Zurich Open Repository and Archive, University of Zurich ZORA URL: https://doi.org/10.5167/uzh-143924

Journal Article

Published Version

Originally published at:

Ruggeri, Francesca; Krishnan, Madhavi (2017). Lattice diffusion of a single molecule in solution. Physical review. E, 96:062406.

DOI: https://doi.org/10.1103/PhysRevE.96.062406 


\title{
Lattice diffusion of a single molecule in solution
}

\author{
Francesca Ruggeri ${ }^{1}$ and Madhavi Krishnan ${ }^{1,2, *}$ \\ ${ }^{1}$ Department of Chemistry, University of Zürich, Winterthurerstrasse 190, CH 8057 Zürich, Switzerland \\ ${ }^{2}$ Department of Physics, University of Zürich,Winterthurerstrasse 190, CH 8057 Zürich, Switzerland
}

(Received 5 September 2017; published 13 December 2017)

\begin{abstract}
The ability to trap a single molecule in an electrostatic potential well in solution has opened up new possibilities for the use of molecular electrical charge to study macromolecular conformation and dynamics at the level of the single entity. Here we study the diffusion of a single macromolecule in a two-dimensional lattice of electrostatic traps in solution. We report the ability to measure both the size and effective electrical charge of a macromolecule by observing single-molecule transport trajectories, typically a few seconds in length, using fluorescence microscopy. While, as shown previously, the time spent by the molecule in a trap is a strong function of its effective charge, we demonstrate here that the average travel time between traps in the landscape yields its hydrodynamic radius. Tailoring the pitch of the lattice thus yields two different experimentally measurable time scales that together uniquely determine both the size and charge of the molecule. Since no information is required on the location of the molecule between consecutive departure and arrival events at lattice sites, the technique is ideally suited to measurements on weakly emitting entities such as single molecules.
\end{abstract}

DOI: 10.1103/PhysRevE.96.062406

\section{INTRODUCTION}

Diffusion in a free-energy landscape is a phenomenon of central importance in condensed matter physics, chemical reactions, and molecular biology. Highly disparate natural phenomena such as atom migration on surfaces or in crystals, molecular chemical transformation, macromolecular interactions, and protein folding can in fact be described in terms of common underlying statistical principles governing transport across energy barriers. Indeed, surface science offers a most tangible example of the phenomenon [1]. Atoms preferentially adsorb at specific sites on the periodic surface lattice which represent locations of the lowest potential energy for adsorption. Driven by thermal energy, adsorbed atoms hop in a random fashion across relatively small energy barriers from one minimum to the next. Depending on the height of the barrier relative to the thermal energy scale, transport of the surface adsorbate can be substantially slowed down or virtually unimpeded as in a fully mobile two-dimensional gas. Direct imaging of atomic and molecular migration using field-ion and scanning-tunneling microscopy has facilitated the measurement of important physical parameters in the transport process such as the activation energy and the attempt frequency. These studies have been instrumental in exploring fundamental features of transition state theory [2-6].

Furthermore, transport in periodic potentials is ubiquitous in biological systems. For example, thermal migration of motor proteins along the periodic interaction energy landscape of a microtubule plays an important role in regulating microtubule length in the cellular cytoskeleton [7]. DNA-binding proteins and enzymes use diffusion along the molecular contour in order to locate specific binding sites. This diffusive search strategy is thought to include a "hopping" mode of transport reminiscent of the classic lattice diffusion problem [8].

Previous experimental studies on particle motion in periodic potentials in the fluid phase have examined diffusive and

\footnotetext{
*madhavi.krishnan@uzh.ch
}

field-driven transport of single colloidal particles and DNA, in spatially modulated gravitational and optical fields, and configurational entropy landscapes [9-13]. Typically these investigations use field-driven transport to separate a mixture of molecular species, exploiting the nonlinear response in object mobility to a spatially varying potential $[14,15]$. However, not much prior effort has been directed at quantitative studies on purely diffusive transport of a single molecule in a well-defined free-energy landscape. A microscopic view of this transport process under well-controlled conditions is not only highly relevant for direct measurements on the properties and interactions of an isolated macromolecule in solution, as we show in our work, but may also contribute toward an improved understanding of molecular transport in naturally occurring modulated systems, e.g., living cells $[16,17]$.

Here we examine diffusive transport of single nanometerscale molecules in a 2D free-energy landscape in the fluid phase. Analogous to the experiments on atom diffusion on a surface, we use optical microscopy to study the thermal migration of a single fluorescently labeled macromolecule in a well-controlled electrostatic interaction free-energy landscape in solution. We further exploit the principles of overdamped transport in a periodic free-energy landscape in order to directly measure the physical properties of a single macromolecule-namely, its size and effective electrical charge in solution.

\section{EXPERIMENTAL METHODS}

\section{A. Experimental design and measurement principle}

In order to create an ordered landscape of potential wells for a single macromolecule, we employ our recently developed thermodynamic approach to trapping electrically charged matter in aqueous solution [18]. The working principle of such a trap is based on the equilibrium repulsive electrostatic interaction between a charged object in solution and likecharged confining parallel plates (Fig. 1). Geometric tailoring of the parallel plates results in a local interaction potential minimum that is capable of confining an electrically charged 


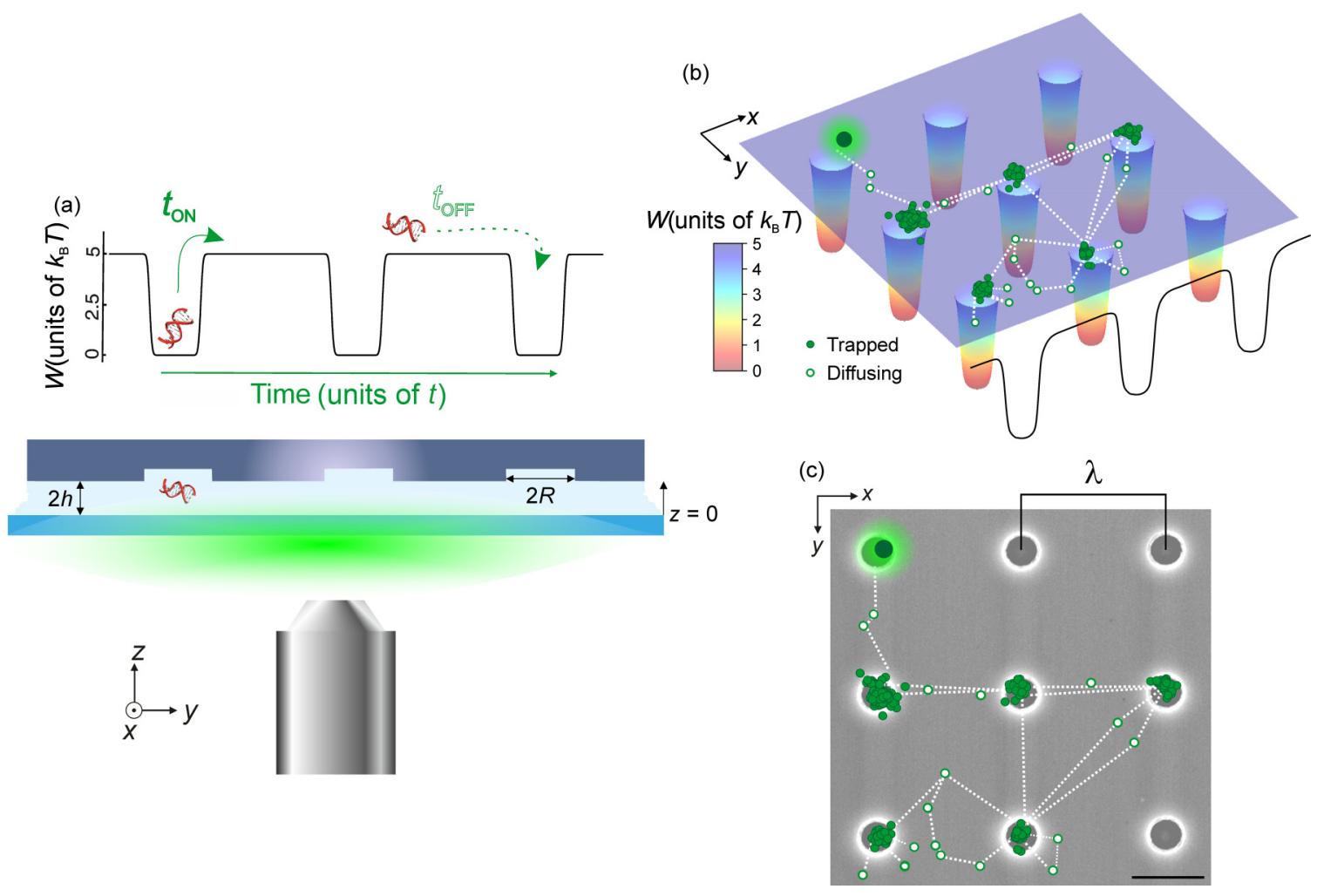

FIG. 1. (a) Schematic representation of the experimental setup displaying a single biomolecule in a lattice of electrostatic traps, observed using wide-field fluorescence microscopy. $2 h$ indicates the slit height while $2 R$ is the diameter of the nanostructured surface indentations that create local electrostatic potential minima. (b) A simulated trajectory of a diffusing molecule superimposed on a representative free-energy landscape, $W(\mathbf{r})$. (c) The same trajectory as in panel (b) superimposed on a scanning electron micrograph (SEM) of the underlying nanostructured surface. Closed symbols denote locations of the particle at lattice sites and open symbols denote free-diffusive transport in the interwell region. $\lambda$ denotes the the lattice pitch. Scale bar denotes $1 \mu \mathrm{m}$.

molecule for long periods ( $\sim 30 \mathrm{~min})$. Molecular residence time scales in a trap can be tuned by the geometry of the trapping nanostructure and salt concentration in solution $[18,19]$.

Briefly, in our experiment, we work with free-energy landscapes created in a gap of typical height, $2 h=73-75 \mathrm{~nm}$, containing surface nanostructured indentations of radius, $R=$ 150 or $300 \mathrm{~nm}$ [Fig. 1(a)] and depth, $150 \mathrm{~nm}$, as previously described [19]. Fluorescently labeled macromolecules or particles in solution are introduced into the lattice at a concentration of $10-50 \mathrm{pM}$ by capillary flow in a buffer containing $1 \mathrm{mM}$ Tris and $0.5-2 \mathrm{mM} \mathrm{NaCl}$ [19]. We studied fluorescent nanoparticles and macromolecules, such as short DNA fragments (10 b ssDNA and 40 and $60 \mathrm{bp}$ dsDNA, purchased from Microsynth AG, Switzerland) as well as an intrinsically disordered starmaker-like protein, Stm-1 [20]. The molecules in our work are labeled with the fluorescent dye Atto532 while the nanoparticles (FluoSpheres, ThermoFisher Scientific) are carboxylated latex spheres labeled with Nile Red. The flow is arrested and molecular motion in the lattice is imaged under purely diffusive conditions by wide-field fluorescence microscopy.

We use optical excitation at $532 \mathrm{~nm}$ from a DPSS laser (Pusch OptoTech GmbH, Germany) and collect fluoresence emission beyond $552 \mathrm{~nm}$. Images of object motion are acquired using a back-illuminated Electron Multiplying CCD
(EMCCD) camera (iXon, Andor Inc., United Kingdom) with continuous exposures of duration $t_{\exp }=5 \mathrm{~ms}$ during a total observation time of about $1 \mathrm{~s}$ per molecular trajectory. The depth of the wells, $W$, in this work is typically $5 k_{\mathrm{B}} T$, yielding trap residence times of $\sim 50 \mathrm{~ms}$. For an object in a potential well in the fluid phase, overdamped diffusive crossing of a barrier is well described by Kramers' theory in the regime $W>6 k_{\mathrm{B}} T$, where the average time to escape the potential well is given by [19]

$$
t_{\mathrm{esc}}=t_{\mathrm{r}} \exp \frac{W}{k_{\mathrm{B}} T} .
$$

Here $t_{\mathrm{r}}$ is a time scale representing the position relaxation time of the molecule, which in turn depends not only on geometric features of the potential well but also importantly on the molecule's diffusion coefficient, $D=k_{\mathrm{B}} T / 6 \pi \eta r_{\mathrm{H}}$. Here, $r_{\mathrm{H}}$ is the hydrodynamic radius of an equivalent sphere that experiences the same frictional drag as the object of interest, and $\eta$ is the viscosity of the medium [21]. Relying on this strong nonlinear dependence, we converted the measured average escape time $t_{\text {esc }}$ of a trapped molecule into a highly precise ( $\sim 1 \%$ ) measurement of the barrier height, $W$ [19]. Note that the barrier height in our case is in essence the interaction free energy of the molecule with the confining parallel-plate slit $[22,23]$. It is also worth emphasizing that this electrostatic interaction energy directly reflects the molecule's effective 
charge, $q_{\text {eff }}$, which is in turn a function not only of its total structural charge but notably also of its three-dimensional (3D) conformation [23].

In our original proof-of-concept electrometry experiment on single molecules, conversion of the measured escape time to a well depth also required accurate knowledge of the free diffusion coefficient or hydrodynamic radius $r_{\mathrm{H}}$ of the molecule [19]. This information was therefore obtained from an independent fluorescence correlation spectroscopy or single-molecule diffusion measurement. Furthermore, although we have shown previously that the electrical charge as well as the size of a particle in an electrostatic potential well can be obtained in a single measurement by, e.g., highprecision tracking of particle position [24] or alternatively by high-temporal resolution optical monitoring of particle fluctuations, doing so typically requires a high signal-to-noise ratio (SNR > 10) in detection, which is generally out of reach in experiments involving weakly emitting single molecules. In addition, the limited photon count rate and overall photon budget would pose a challenge for precise measurements on molecular-scale entities where the position fluctuation dynamics can be up to two orders of magnitude faster than for nanoparticles.

We emphasize that in this work the Debye length, $\kappa^{-1} \approx$ $0.3 / \sqrt{C}=10 \mathrm{~nm}$, is the characteristic length scale of screening of electric fields in an electrolyte containing monovalent salt at a concentration, $C=10^{-3} \mathrm{~mol} / \mathrm{L}$. Since in these experiments, the lattice pitch, $\lambda \sim 300 \kappa^{-1}$ [Fig. 1(c)], the interwell region of the landscape, is free of electrical fields in the $x y$ plane and the in-plane transport of the molecule should be well described by free diffusion in two dimensions. We show later using validation experiments on nanoparticles that this is indeed the case. There are, however, strong axial forces (in $z$ ) in these regions which keep the particle tightly confined to the midplane of the slit. The solution-phase two-dimensional (2D) free-energy landscapes in our present work therefore differ significantly from those encountered in surface-atom diffusion in that in our case the wells are local regions of potential energy minima in an otherwise curvature-free energy landscape [Figs. 1(b) and 2(a)].

Crucially, such a design permits us to observe molecular transport in both the trapped and freely diffusive regimes.

Monitoring the thermal migration of a single macromolecule in the lattice, we indeed observe two different regimes in molecular transport: a trapped state and a freediffusive state that alternate in time and whose durations can be readily measured. The acquired images of lattice migration are analyzed using an intensity threshold to yield "on times," $t_{\mathrm{ON}}$, where the molecule is confined at a trap location and a large signal accumulates locally on the detector (the same as $t_{\text {esc }}$ in our previous work), and "off-times," $t_{\mathrm{OFF}}$, where it performs free diffusion traveling from one trap to the next and no substantial signal is received [Fig. 3(b)]. Comparing measurements of these two time scales with the predictions of a Brownian dynamics (BD) simulation, we demonstrate the ability to extract information on both the size and effective charge of the molecule from a single transport trajectory. Interestingly, the hopping motion of a molecule in the lattice yields a telegraphic on-off signal in optical detection which permits

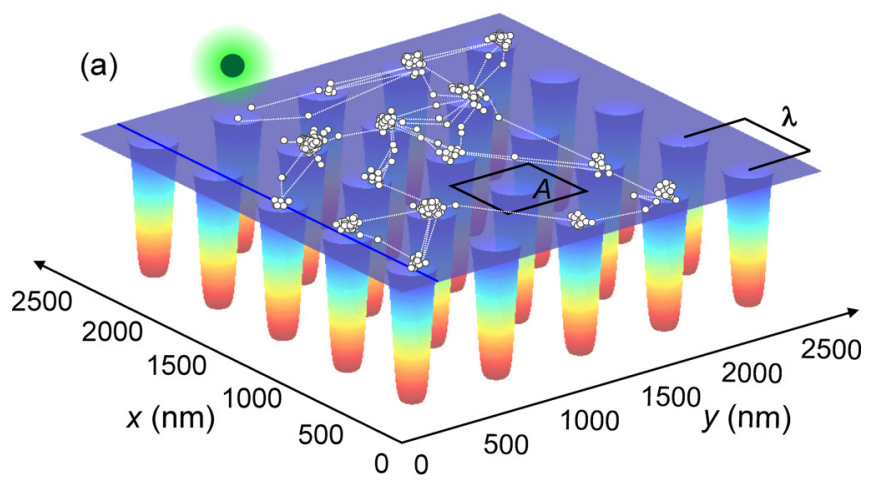

(b)

$W\left(k_{\mathrm{B}} T\right)$

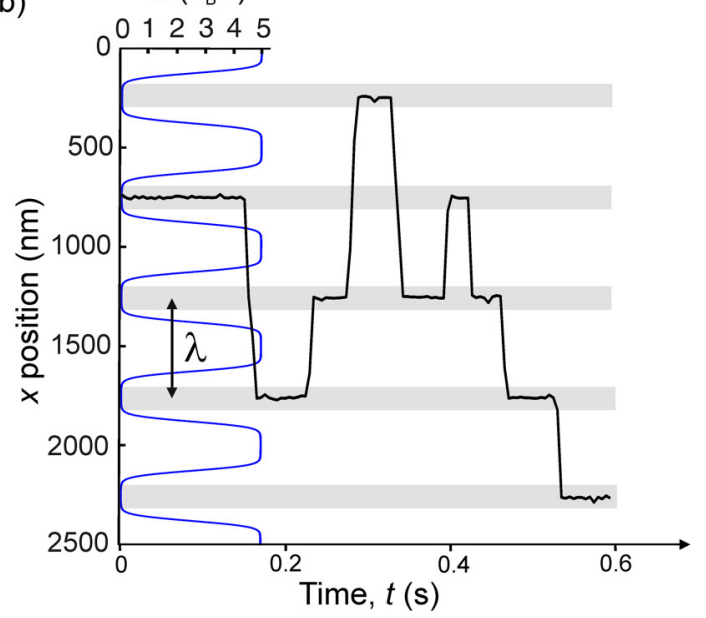

(c)

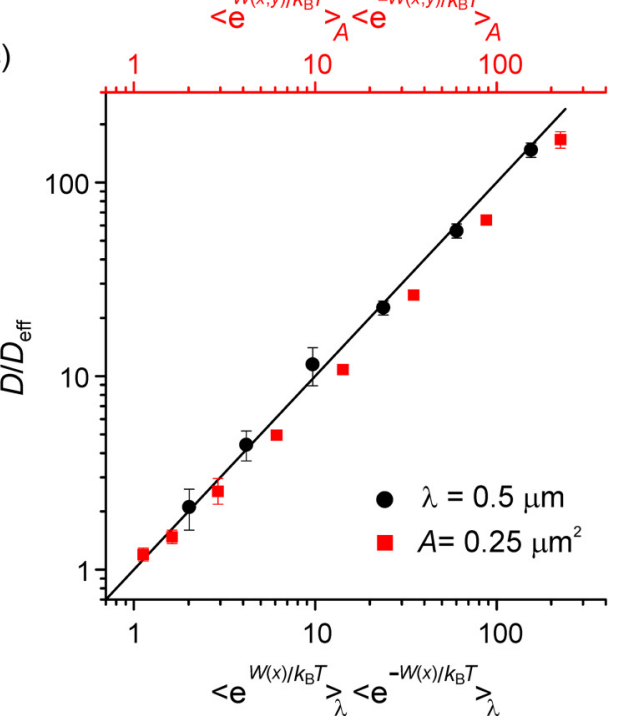

FIG. 2. (a) A simulated single-molecule trajectory superimposed on a two-dimensional lattice of electrostatic traps. $\lambda$ denotes the lattice pitch, while $A$ demarcates a single unit cell. (b) A simulated diffusive trajectory (black trace) in a one-dimensional periodic potential (blue vertical trace) along the solid blue line shown in panel (a). Shaded gray regions depict the part of molecular trajectory confined to the bottom of a given well. (c) The effective diffusion coefficient, $D_{\text {eff }}$, extracted from a mean-squared-displacement (MSD) analysis of simulated trajectories in both one (black circles, bottom axis) and two dimensions (red squares, top axis). The resulting $D / D_{\text {eff }}$ values compare with the Lifson-Jackson expression, Eq. (2) (solid black line). 
(a)
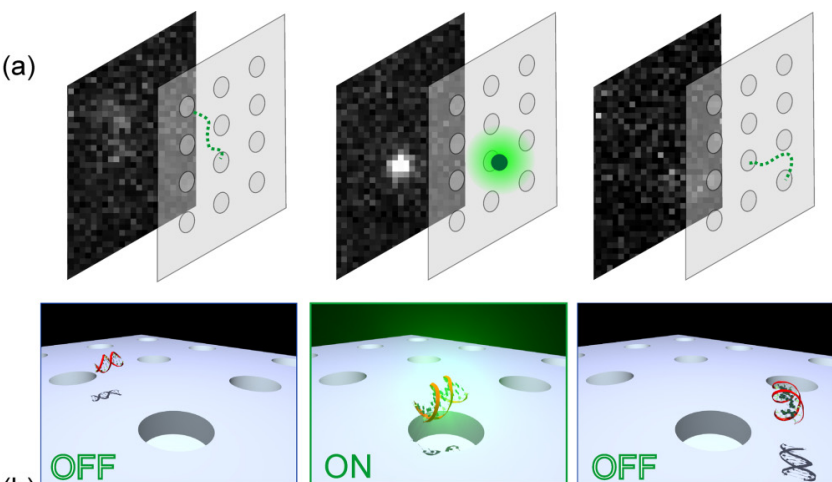

(b)
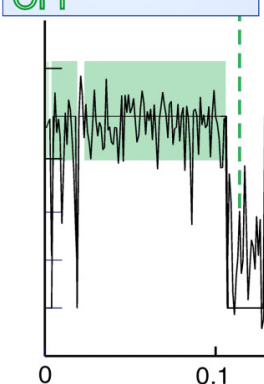
ON
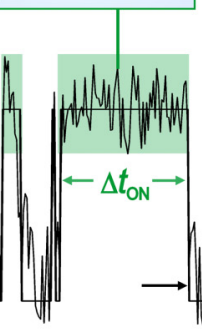
OFF

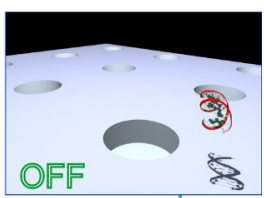

0

0.1 Time, $t(\mathrm{~s})$

(c)

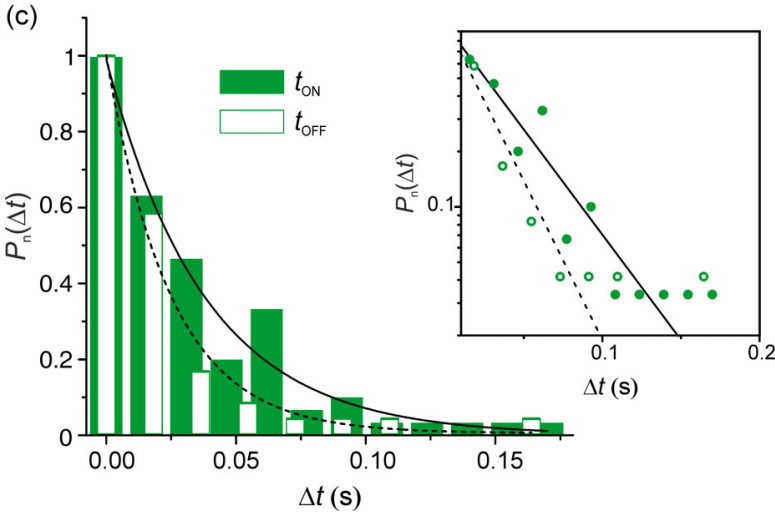

FIG. 3. (a) Experimental raw fluorescence images and corresponding schematic representations depicting a single $60 \mathrm{bp}$ dsDNA molecule sampling a lattice of traps. (b) A simulated temporal trace of the total optical intensity from the lattice sites. The signal is high for a duration $\Delta t_{\mathrm{ON}}$ when the molecule is trapped at a lattice site and sufficient photons accumulate locally on the detector (green bands); the signal is low for a period $\Delta t_{\mathrm{OFF}}$ during free diffusion of the molecule between lattice sites. (c) Probability density distributions, $P(\Delta t)=\frac{A_{\mathrm{fit}}}{t} \exp (-\Delta t / t)$, of experimentally recorded times $\Delta t_{\mathrm{OFF}}$ (white bars) and $\Delta t_{\mathrm{ON}}$ (green bars), for a single trajectory of a 60 bp dsDNA molecule, where $A_{\mathrm{fit}} \approx 1$. The distributions are normalized such that $P_{\mathrm{n}}(\Delta t)=\frac{P(\Delta t)}{P_{\max }(\Delta t)}$. The fits yield average values $t_{\mathrm{OFF}}=25 \pm 2 \mathrm{~ms}$ and $t_{\mathrm{ON}}=38 \pm 4 \mathrm{~ms}$.

precise measurements to be performed at very low SNR $(\sim 3)$ [Fig. 3(a) and Fig. 12 in Appendix B]. We thus introduce a lattice-diffusion principle that is ideally suited to measurements on weakly emitting single macromolecules in solution.

\section{B. Interpreting the detected single-molecule signal}

We define the signal received from the molecule as the difference between the total optical intensity due to the
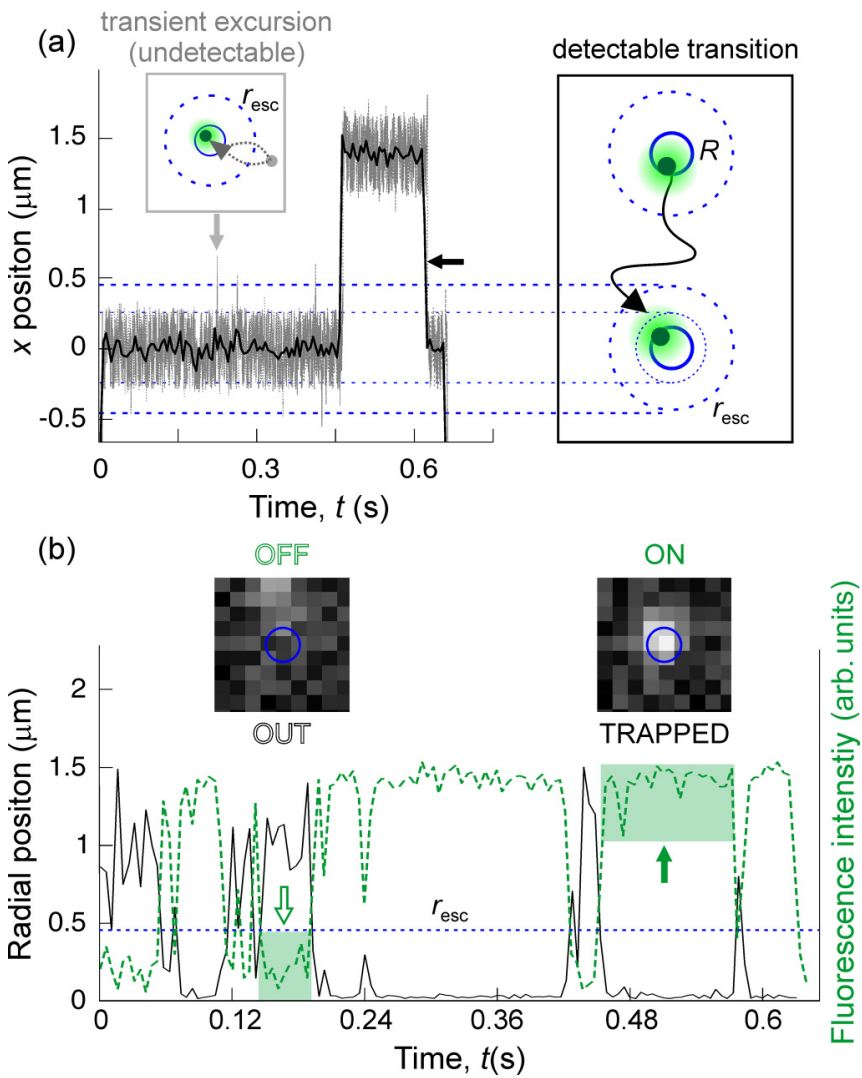

FIG. 4. (a) A simulated particle trajectory at a time resolution of $1 \mu \mathrm{s}$ (gray dashed line), time averaged to a resolution of $5 \mathrm{~ms}$ (black solid line) in order to reflect experiments where the exposure time $t_{\text {exp }}=5 \mathrm{~ms}$. The accompanying schematics depict the circumference of the surface indentations (solid blue lines) and various escape boundaries (dotted blue lines). We highlight transient rapid excursions of the molecule out of and back into the trap which go undetected (light gray arrow), as well as transitions of the molecule from one trap to the next which are detected in the simulation and experiment as a "hop" between lattice sites (black arrow). (b) Generation of a series of optical images corresponding to molecular coordinates in a trajectory (black trace, left axis) shows that the signal from the lattice is high when a molecule is trapped at a lattice site and low otherwise (green dashed trace, right axis). The location of the escape boundary, $r_{\mathrm{esc}}$, applied to the simulated trajectory data is tuned such that the resulting on and off-time scales agree with those from the simulated optical signal. These time scales are then compared with the corresponding experimental measurements.

molecule and that due to the detector background. When a weakly emitting entity occupies a potential well, its radius of spatial confinement ( $\sim 250 \mathrm{~nm})$ is comparable with the optical point spread function. As a result, a measurable local signal builds up on a spatially sensitive detector such as a camera. In contrast, when the molecule leaves a well and diffuses in the "field-free" interwell zone of the landscape, the received signal does not exceed the local detector background and therefore gives rise to a "dark time" during the measurement, which we term $t_{\mathrm{OFF}}$ (see the movie in the Supplemental Material [25]).

Analysis of the detected optical signal from a single molecule diffusing in a 2D lattice yields the two average time scales: an on time, $t_{\mathrm{ON}}$, and an off-time, $t_{\mathrm{OFF}}$ [Figs. 3(a) 


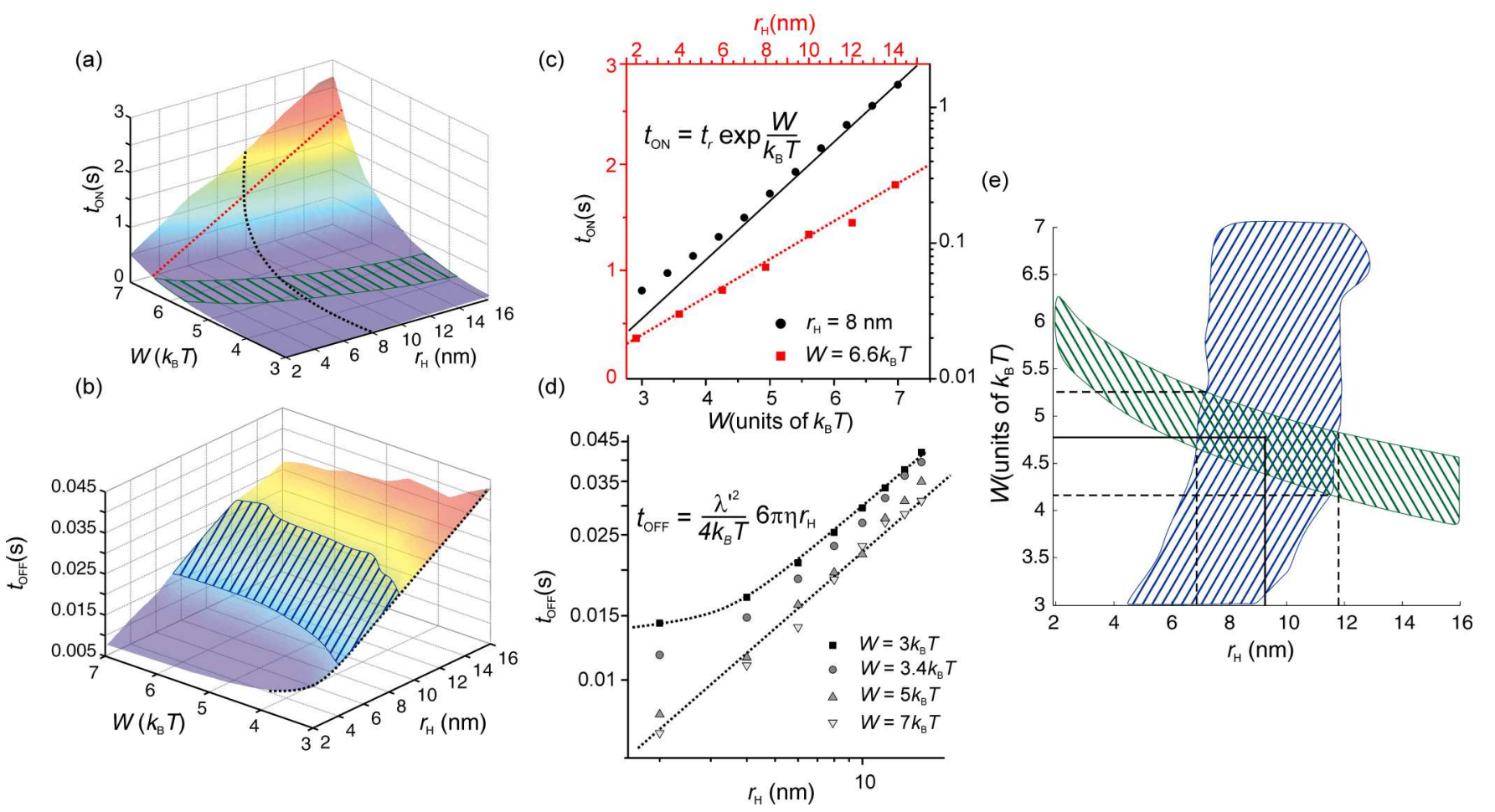

FIG. 5. [(a), (b)] Simulated $t_{\mathrm{ON}}$ and $t_{\mathrm{OFF}}$ values for relevant combinations of $W$ and $r_{\mathrm{H}}$. (c) Plots of $t_{\mathrm{ON}} \mathrm{vs} W$ at $r_{\mathrm{H}}=8 \mathrm{~nm}$ (black circles, bottom and right axis) and $t_{\mathrm{ON}}$ vs $r_{\mathrm{H}}$ for $W=6.6 k_{\mathrm{B}} T$ (red squares, top and left axis) along the contour lines shown in panel (a). $t_{\mathrm{ON}}$ depends linearly on $r_{\mathrm{H}}$ but is exponential in $W$. (d) $t_{\mathrm{OFF}}$ vs $r_{\mathrm{H}}$ for various values of $W$. The relationship is linear in the high well-depth regime ( $W>5 k_{\mathrm{B}} T$ ), but starts to plateau for low $r_{\mathrm{H}}$ and $W<6 k_{\mathrm{B}} T$. Dashed lines are guides to the eye. (e) The hatched regions on the plots in panels (a) and (b) depict combinations of $W$ and $r_{\mathrm{H}}$ that satisfy a measured $t_{\mathrm{ON}}$ and $t_{\mathrm{OFF}}$ value independently, including measurement uncertainty (here shown for a single Stm-1 molecule of $t_{\mathrm{ON}}=0.2 \pm 0.065 \mathrm{~s}$ and $t_{\mathrm{OFF}}=0.023 \pm 0.005 \mathrm{~s}$ ). The intersection of the set of solutions for both time scales yields mean measured values of $r_{\mathrm{H}}$ and $W$ (and thus $q_{\mathrm{m}}$ ) (solid black lines). The statistical error on the measurements are given by the lateral and vertical extents of the hatched regions (dashed black lines) (see Appendix D).

and 4(b)]. Similar to memoryless escape from a potential well, we find that, as expected, off-times are also exponentially distributed [Fig. 3(c)]. Importantly, the mean off-time, $t_{\mathrm{OFF}}$, depends linearly on the molecular hydrodynamic radius, $r_{\mathrm{H}}$, while $t_{\mathrm{ON}}$ depends exponentially on the effective charge of the molecule and relatively weakly on $r_{\mathrm{H}}$ [Fig. 5(c)]. A BD simulation of the hopping process yields $t_{\mathrm{ON}}$ and $t_{\mathrm{OFF}}$ times over the $\left\{W\left(q_{\text {eff }}\right), r_{\mathrm{H}}\right\}$ space of interest and thereby serves to convert an experimental measurement of the two measured time scales into unique values of $q_{\mathrm{m}}$ and $r_{\mathrm{H}}$, with respective uncertainties for a single molecule.

\section{BROWNIAN DYNAMICS SIMULATION OF PARTICLE DIFFUSION IN A FREE-ENERGY LANDSCAPE}

Analytical expressions for the average escape time for a particle in a potential well, or transport coefficients in a landscape, are generally only available for particular analytical functional forms of the underlying potential and entail landscapes of infinite extent $[21,26,27]$. As escape times do depend on subtle features such as local curvatures of the landscape, e.g., at the bottom of the well and at the barrier [28], and our experiments involve lattices of finite extent in both dimensions, we performed BD simulations of particle motion in periodic landscapes in order to extract quantitative predictions that accurately reflect our experiments.

\section{A. Generating the free-energy landscape}

The first step in the simulation study is to determine the full three-dimensional distribution of electrical potential in a single well by solving the nonlinear Poisson-Boltzmann (PB) equation in the fluidic trapping nanostructure [18]. We have verified that in our work, the spatial electrostatic free energy for a charged object can be simply obtained by multiplying the local electrical potential $\psi(\mathbf{r})$ by a parameter $q_{\mathrm{eff}}$, which is the effective charge of the object [23]. We point out that since the position of the particle is strongly weighted toward the local minimum of interaction energy, the electrical potential in the region of the midplane of the slit largely determines the overall behavior. Thus, the electrostatic free energy, which is the dominant contribution to $W$ in this work, is well estimated by $q_{\mathrm{eff}} \psi_{\mathrm{m}}$, where $\psi_{\mathrm{m}}$ is the electrical potential at the midplane of the slit.

Furthermore, the problem has a free-energy contribution of typically $30 \%$ from particle spatial fluctuations in the axial $(z)$ dimension. We have verified by simulation that the dimensionality of the problem can be reduced to two by including an entropic correction for axial fluctuations both inside and outside the trap, and an additional $\sim 6 \%$ contribution to the electrostatic energy arising from axial fluctuations in the slit region [19]. Thus, the depth, $W$, of each well in the landscape can be expressed as $W=q_{\mathrm{eff}} \psi_{\mathrm{m}}+f$, where the first term is due to the electrostatic interaction free energy of 
a particle at the midplane of the slit and $f$ is the contribution to the total free energy from the finite out-of-plane thermal fluctuations of the particle. Note that in formulating the well depth, $W$, we deliberately ignore the system free energy when the particle is at the bottom of the well, as the electrical potential here is zero by design.

The geometry of a single potential well is further influenced by the diameter of the surface nanostructure, height of the slit, and the salt concentration in solution, which are all included in the PB calculation. We thus obtain the free-energy landscape for a single primitive cell in the lattice which we then use to generate a 2D landscape of the required lateral pitch and spatial extent in $x$ and $y$ for the simulation [Fig. 2(a)].

\section{B. Effective diffusion coefficient of a particle in the lattice}

The experiments we report here involve a square or rectangular lattice of effectively identical radially symmetric potential wells of depth, $W$, with each well occupying a single lattice site. Analytical treatments of diffusive transport in an infinite one-dimensional periodic arrangement of wells show that the effective diffusion coefficient, $D_{\text {eff }}$, of a molecule strongly depends on $W$ and in the overdamped limit is given by the Lifson-Jackson relation [29-32]:

$$
D=D_{\text {eff }}\left\langle\exp \frac{W(x)}{k_{\mathrm{B}} T}\right\rangle_{\lambda}\left\langle\exp \frac{-W(x)}{k_{\mathrm{B}} T}\right\rangle_{\lambda},
$$

where $D$ is the particle's free diffusion coefficient.

We use the effective diffusion coefficient as the starting point of our analysis of single-molecule transport in a periodic potential. We iteratively solve the discretized overdamped Langevin equation for particle motion in a 2D free-energy landscape of "infinite" extent:

$$
\mathbf{x}(t+\delta t)=\mathbf{x}(t)-\mu \nabla W[\mathbf{x}(t)]+\sqrt{2 D \Delta t} \mathbf{w}(t) .
$$

Here, $\mathbf{x}(t)$ represents the instantaneous position of a particle at time, $t$, in one dimension, and $\mu=\delta t / 6 \pi \eta r_{\mathrm{H}} . \mathbf{w}(t)$ represents a displacement due to the random thermal force acting on the particle that satisfies $\langle\mathbf{w}(t)\rangle=\mathbf{0}$ and $\left\langle\mathbf{w}(t) \mathbf{w}^{\top}\left(t^{\prime}\right)\right\rangle=I$ if $\left|t-t^{\prime}\right| \leqslant \delta t$, and $\mathbf{0}$ otherwise. Note that the simulation time step, $\delta t=10 \mu \mathrm{s}$, is much larger than the momentum relaxation time, which is $\sim 10 \mathrm{~ns}$ for a typical molecule.

At $t=0$, the particle is located at the center of a trap in the landscape. We then use Eq. (3) to propagate the instantaneous position of the particle, $\mathbf{x}(t)$, forward in time over a typical observation period of $30 \mathrm{~s}$. A typical such trajectory in one dimension, time averaged over the exposure time $t_{\exp }$, is shown in Fig. 2(b). In order to validate our simulations against theoretical expectations, we apply a mean-squared-displacement (MSD) analysis to about five particle trajectories and extract effective lattice diffusion coefficients, $D_{\text {eff }}$, for the particle. We verify that the simulated $D_{\text {eff }}$ values satisfy the Lifson-Jackson relation in both one and two dimensions for values of $W$ ranging from 3 to $8 k_{\mathrm{B}} T$ [29,33] [Fig. 2(c)]. Experiments on micron-scale particles in a periodic landscape have verified the Lifson-Jackson relation [34] and demonstrated a measurement of the effective diffusion coefficient, $D_{\text {eff }}$, of a particle [9]. However, knowledge of $D_{\text {eff }}$ alone yields neither the well depth, $W$, nor the particle's free diffusion coefficient, $D$, which reflects its hydrodynamic radius, $r_{\mathrm{H}}$.

\section{Trapped and free diffusive-state lifetimes for a single particle}

Our study requires us to go beyond the analysis of an effective diffusion coefficient for molecular transport in a lattice. Our goal is to accurately determine both the trapped and free diffusive time scales for a single particle migrating in a periodic lattice in order to obtain measures for both $W$ and $D\left(r_{\mathrm{H}}\right)$ from a single transport trajectory. A recent study on colloidal particle transport in a quasicrystalline lattice illustrates the subtleties involved and shows how the lack of crystalline order in the landscape introduces more complex free diffusive behavior, thus necessitating averaging over an ensemble of particles in order to measure both $W$ and $r_{\mathrm{H}}$ [34].

In order to correctly relate the time scales of interest observed in experiment to those available from simulations, we perform further analysis of the simulated trajectories involving important additional features. One such feature is the definition of an absorbing "escape boundary" represented by a radially symmetric contour at $r_{\text {esc }}$ centered on the trap [Fig. 4(a)].

When analyzing a simulated transport trajectory of an object diffusing in the landscape, we register a trapped state of duration $\Delta t_{\mathrm{ON}}$ when the particle enters the region within the predefined domain circumscribed by $r_{\text {esc }}$ at time $t$, then reaches the bottom of the trap - denoted by a circular region of radius, $R / 2$ centered on the trap - and subsequenty leaves the domain given by $r_{\mathrm{esc}}$ at a time $t^{\prime}>t$. Here $\Delta t_{\mathrm{ON}}=t^{\prime}-t$. $\Delta t_{\mathrm{OFF}}$ is then the interval between two sequential on-states. In addition, since the imaging process involves a finite time window of observation, the experimentally measured particle position in fact physically reflects its location averaged over the duration of an exposure time $\langle r\rangle_{t_{\text {exp }}}$, rather than its instantaneous position, $r$. Furthermore, in the escape-froma-potential well problem, it is well known that the location of the absorbing boundary condition can strongly influence the value of the average escape time or the trapped state lifetime [35] (Appendix A). Simulation results presented in Fig. 4(a) illustrate two important features: (1) The average escape time of the instantaneous position, $r$, depends strongly on the location of the escape boundary, and (2) escape times based on a time-averaged position, $\langle r\rangle_{t_{\mathrm{exp}}}$, should be substantially longer than that based on an instantaneous position, $r$.

We analyze via simulation the dependence of $t_{\mathrm{ON}}$ on $r_{\mathrm{esc}}$ the location of the escape boundary (Fig. 11 in Appendix A). Given the average diffusion length during an exposure time of duration, $t_{\exp }$, is $l_{\text {diff }}=\sqrt{4 D t_{\text {exp }}}$, we find that for $\lambda<l_{\text {diff }}$, the measured time scale $t_{\mathrm{ON}}$ does not strongly depend on the location of the boundary. This is because the proximity of traps ensures that escape from one well is highly likely to result in absorption at the neighboring lattice site. When $\lambda>l_{\text {diff }}$, however, as in these experiments reported here, the simulated escape time, $t_{\mathrm{ON}}$, does strongly depend on the location of the escape boundary (see Fig. 11 in Appendix A). This is due to the fact that in this regime a particle that escapes a given well and crosses $r_{\text {esc }}$ has, on short time scales, a finite probability of return to the same well rather than diffusion to and capture at the neighboring well [34].

Furthermore, as already described, the experimental readout in the case of single molecules is not a temporally varying particle position in the lattice but rather a high or low optical signal at the lattice sites [Fig. 3(a)]. It is therefore essential to determine the true location of the absorbing boundary 
condition in the simulation that would correctly reflect the optical on-off time scales measured in experiments. In addition, other experimental factors such as SNR and detector background could also influence the measurement of the time scales of interest. Since time averaging and subtle features of the optical detection described above make it a priori unclear where to place the escape boundary in the particle-coordinate output of the BD simulation, $r_{\mathrm{esc}}$ has to be obtained by matching particle position-based predictions of the on-off time scales against those from simulated optical signal data.

We therefore also perform an image-based analysis of simulated thermal migration of the molecule in the lattice including most, if not all, of the important attributes of the imaging process and system, e.g., exposure time, typical signal and background intensities, background noise, and the optical point spread function [Fig. 4(b)]. These simulated images of molecular transport are generated in an additional step after the BD simulation, using trajectories generated for various values of particle size and charge. An example of a particle position trace and the corresponding optical signal are shown in Fig. 4(b).

The optical traces are analyzed in terms of intensity thresholds on the signal, in a manner identical to the experimental data, to yield values of $t_{\mathrm{OFF}}$ and $t_{\mathrm{ON}}$ for a single hopping trajectory about $30 \mathrm{~s}$ in length. Importantly, the time scales obtained from this full image-based analysis can be compared with those from the corresponding "raw" particle coordinates in order to determine the location of the escape boundary, $r_{\mathrm{esc}}$, that accurately recovers the same $t_{\mathrm{ON}}$ and $t_{\mathrm{OFF}}$ values [Fig. 4(b)]. As a general rule for the lattices in these experiments, we find that a radially symmetric absorbing boundary located at $r_{\text {esc }}=R+300 \mathrm{~nm}$ yields values of $t_{\mathrm{OFF}}$ and $t_{\mathrm{ON}}$ within $10 \%$ of the full image-based analysis. The advantage of working with simulations based on the coordinates of the particle, $\langle r\rangle_{t_{\text {exp }}}$, rather than detected optical images is that the former approach is computationally less demanding and faster than the image-based route.

Interestingly, we find that for strongly emitting objects (photon count rate $>10 \mathrm{kHz}$ ), where particle motion can be directly tracked at all times, self-consistent results are obtained as long as the experimentally measured position traces are analyzed using the same escape boundary criterion as the simulated traces, regardless of the actual location of the boundary.

As $t_{\mathrm{OFF}}$ and $t_{\mathrm{ON}}$ each depend on both $q_{\mathrm{eff}}$ and $r_{\mathrm{H}}$, the simulation analysis yields two surface plots for the time scales as a function of the two measurables of interest (Fig. 5). We note that $t_{\mathrm{OFF}}$ not only depends linearly on the molecular hydrodynamic radius, $r_{\mathrm{H}}$, but also depends strongly on geometric parameters such as the lattice pitch, $\lambda$, and further displays a weak dependence on the depth of the traps, $W$ [Fig. 5(d)]. $t_{\mathrm{ON}}$ in turn, as previously shown, depends exponentially on $W$ (or $q_{\text {eff }}$ ) and only linearly on $r_{\mathrm{H}}$ [Fig. 5(c)]. The simulation result thus serves to convert an experimental measurement of the two time scales $t_{\mathrm{ON}}$ and $t_{\mathrm{OFF}}$ into unique values of the two unknowns, namely the measured effective charge, $q_{\mathrm{m}}$, and hydrodynamic radius, $r_{\mathrm{H}}$, with respective uncertainties for a single molecule.

Finally, we point out that the precision on the inferred value of $q_{\mathrm{m}}$ is ultimately limited by the statistical uncertainty inherent to determining the diffusion coefficient, $D$, of a single molecule from a temporally limited migration trajectory in the lattice. Measurement precision improves with

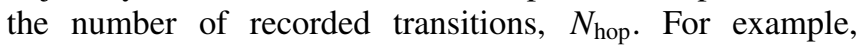
$N_{\text {hop }}=100$ in a measurement with $t_{\mathrm{OFF}}=t_{\mathrm{ON}}=$ $25 \mathrm{~ms}$ implies precision of $9 \%$ and $17 \%$ in the charge and size measurements, respectively, in a total measurement time of $\sim 2 \mathrm{~s}$ (see Appendix D). Armed with a full-fledged simulation-based quantitative analysis of the problem, we then proceed to an experimental validation of the predictions as well as measurements of the properties of single molecules.

\section{EXPERIMENTAL VALIDATION USING FLUORESCENT NANOSPHERES}

The simulations reveal a linear dependence of $t_{\mathrm{OFF}}$ on $r_{\mathrm{H}}$ in the large- $W$ regime, reflecting the fact that $t_{\mathrm{OFF}}$ is nothing but the average diffusion time of the object over a distance given by an effective lattice pitch, $\lambda^{\prime}$ [Fig. 6(a)]. In order to experimentally test the accuracy of the relationship between $t_{\mathrm{OFF}}$ and $r_{\mathrm{H}}$ predicted by the simulation, we performed measurements of nanoparticle diffusion in a free-energy landscapes created in slits of height $2 h=200 \mathrm{~nm}$.

In contrast to molecules, nanoparticles emit strong signals in optical excitation and can therefore be spatially tracked with high precision during the entire transport trajectory. A meansquared-displacement (MSD) analysis of nanosphere motion in the free-diffusive regime directly yields its hydrodynamic radius, $r_{\mathrm{H}}$. The same data can also be used to extract average interwell travel times, which we have referred to as $t_{\mathrm{OFF}}$ for a weakly emitting molecule. Thus we compare the value of $r_{\mathrm{H}}$ obtained from $t_{\mathrm{OFF}}$ measurements in a given lattice, with a parameter-free, direct determination of the same quantity in the same measurement via the MSD approach and find good agreement [Fig. 6(d)]. This result validates our $r_{\mathrm{H}}$ measurement principle and warrants its further application to measurements on single molecules. Note that at a particle diameter to slit height ratio of $a / h \simeq 0.2$ the viscous drag on the particle is expected to increase by $20 \%$ [37,38]. The slightly inflated value of particle radius, $r_{\mathrm{H}}$, obtained in the lattice MSD measurement compared to bulk measurements is in fact in line with this expectation.

For the measurements on macromolecules, described in the following section, assuming the molecules behave as hard spheres of radius, $r_{\mathrm{H}}$, we expect drag enhancement factors due to confinement ranging from $6 \%$ for 10 base DNA to $23 \%$ for the disordered protein Stm-1 [39]. The measurement averages for $r_{\mathrm{H}}$ (Table I), however, reveal no systematic departure from the free solution measurements, suggesting that the true reduction in diffusion coefficient is probably much smaller than the above estimates for hard spheres.

\section{MEASUREMENTS ON SINGLE MACROMOLECULES IN SOLUTION}

We now focus on measurements of effective charge and hydrodynamic radius on single macromolecules in solution. We have verified using simulations that quantitatively identical escape times are obtained by replacing the spatial electrical potential distribution in the slit from the nonlinear PB equation by an axial potential given by the superposition of two simple 
(a)
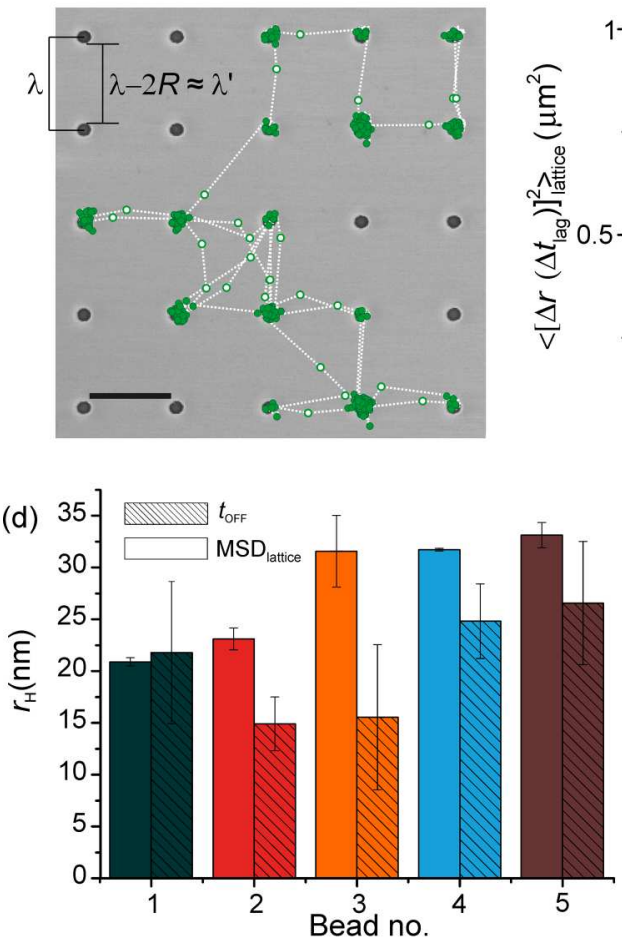

(b)

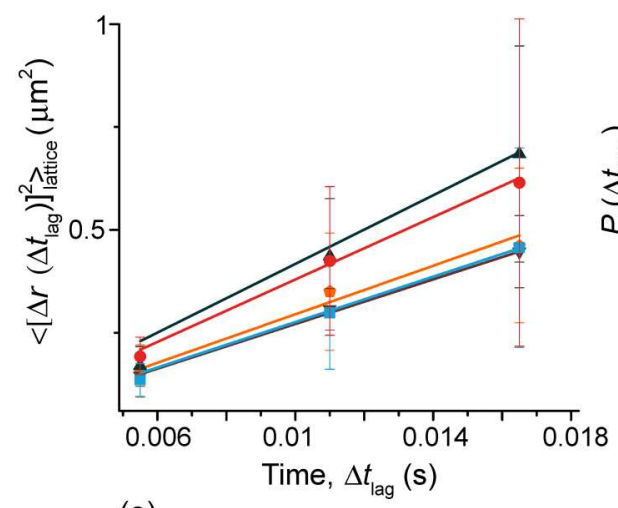

(e)

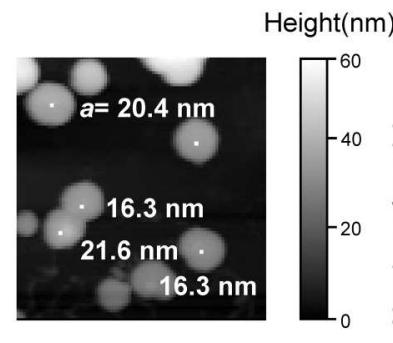

(c)

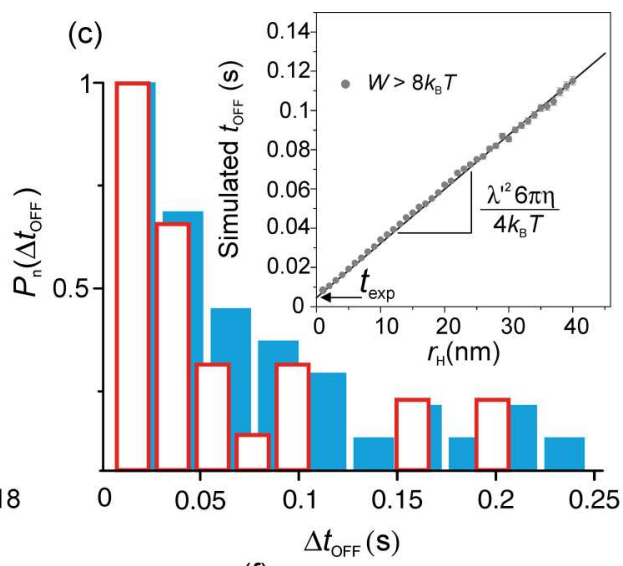

(f)

\begin{tabular}{cc} 
Method & $\begin{array}{c}\text { Radius } \\
\text { (nm) }\end{array}$ \\
\hline AFM & $24.8 \pm 4$ \\
DLS & $23.4 \pm 10$ \\
MSD (free) & $24.1 \pm 1.3$ \\
MSD (lattice) & $28 \pm 5$ \\
$\boldsymbol{t}_{\text {OFF }}$ & $20.9 \pm 4.8$ \\
\hline
\end{tabular}

FIG. 6. (a) A representative trajectory of a fluorescent nanosphere of nominal radius $a=24 \mathrm{~nm}$ diffusing in a square lattice of pitch, $\lambda=2 \mu \mathrm{m}$ and $R=150 \mathrm{~nm}$, overlaid on an SEM image of the nanostructured surface. The effective pitch $\lambda^{\prime}$ inferred from the fit in panel (c) is close to the value $\lambda-2 R$, which corresponds to the edge-to-edge distance of two neighboring traps. The trapped (solid green symbols) and free-diffusive regimes (open symbols) of the particle trajectory are identified. Scale bar denotes $2 \mu \mathrm{m}$. (b) Single-particle trajectories in the free-diffusive regime (open symbols) are used to evaluate the mean-squared displacement (MSD), $\Delta \mathbf{r}\left(\Delta t_{\mathrm{lag}}\right)$, as a function of lag time, $\Delta t_{\mathrm{lag}}$, in two dimensions. A fit of the form $\left\langle\left[\left(\Delta \mathbf{r}\left(\Delta t_{\mathrm{lag}}\right)\right]^{2}\right\rangle=4 D \Delta t_{\mathrm{lag}}+\epsilon^{2}\right.$, where $\epsilon$ represents the particle localization uncertainty, yields $D$ [36]. Here, $\Delta t_{\mathrm{lag}}=5.5 \mathrm{~ms}$ and each symbol represents a different nanosphere. (c) Normalized probabilty distributions $P_{\mathrm{n}}\left(\Delta t_{\mathrm{OFF}}\right)$ are presented for two particles in panel (b). The open bars correspond to circular symbols and solid bars to square symbols in panel (b). The simulated $t_{\mathrm{OFF}} \mathrm{vs} r_{\mathrm{H}}$ is linear in the large $W$ regime (inset, gray symbols). The intercept of the linear fit (black line) corresponds to the exposure time, $t_{\text {exp }}$, used in the simulation and experiment, while the slope, as expected, carries information on the effective pitch $\lambda^{\prime}$. Here we find from the experimental dataset $\lambda^{\prime}=1.6 \pm 0.01 \mu \mathrm{m}$. (d) Both $\mathrm{MSD}_{\text {lattice }}$ and $t_{\mathrm{OFF}}$ approaches yield independent measures for $r_{\mathrm{H}}$ for individual particles that are in good agreement. Data for individual nanospheres (left to right) correspond to the data series in panel (b) from top to bottom. (e) The hard sphere radius of the beads was characterized via atomic force microscopy (AFM). The height of a sample of spheres spin coated on a glass slide averaged over 90 particles agrees well with the nomimal diameter of the sample. (f) Comparison of size values measured by different approaches including dynamic light scattering (DLS) are in excellent mutual agreement. Please note that while AFM measurements provide information on the hard sphere radius of a particle, the other four approaches directly measure $r_{\mathrm{H}}$.

TABLE I. Measurements of hydrodynamic radius, $r_{\mathrm{H}}$, and effective charge, $q_{\mathrm{m}}$, averaged over all single-molecule measurements reported for each species in Fig. 7. For comparison, we include independent $r_{\mathrm{H}}$ measurements using ensemble-averaging techniques, and theoretically expected values of effective charge, $q_{\mathrm{c}}$, including the contribution of the dyes.

\begin{tabular}{|c|c|c|c|c|}
\hline & \multicolumn{2}{|c|}{$\begin{array}{c}r_{\mathrm{H}} \\
(\mathrm{nm})\end{array}$} & \multirow{2}{*}{$\begin{array}{c}q_{\mathrm{c}} \\
(-e)\end{array}$} & \multirow{2}{*}{$\begin{array}{c}q_{\mathrm{m}} \\
(-e)\end{array}$} \\
\hline & FCS/DLS & $t_{\mathrm{OFF}}$ & & \\
\hline Nanospheres & $23.4 \pm 10$ & $20.9 \pm 4.8$ & & $181.7 \pm 12.6$ \\
\hline Stm-1 & $8.7 \pm 0.5$ & $7.9 \pm 2.1$ & 89.6 & $105.5 \pm 21.6$ \\
\hline $60 \mathrm{bp}$ & $4.5 \pm 0.2$ & $4.7 \pm 0.9$ & 43.4 & $37.9 \pm 4$ \\
\hline $40 \mathrm{bp}$ & $3.2 \pm 0.2$ & $2.4 \pm 0.4$ & 32.7 & $32.1 \pm 5.1$ \\
\hline 10 base & $1.8 \pm 0.1$ & $1.5 \pm 0.8$ & 10.9 & $8.3 \pm 1.9$ \\
\hline
\end{tabular}

exponentials of the form

$$
\psi(z)=\psi_{\mathrm{s}}\{\exp (-\kappa z)+\exp [-\kappa(2 h-z)]\}
$$

where $\psi_{\mathrm{s}}$ is an effective surface potential at each surface of the slit and $2 h$ is the height of the slit. This approximation works well because the Boltzmann distribution ensures that the molecule essentially never samples the region very close to the surfaces, where differences in the electrical potential between the nonlinear PB solution and the equivalent linear theory could arise.

We know from our previous measurements in this system that setting $\psi_{\mathrm{s}}=2.8 k_{\mathrm{B}} T / e$ in Eq. (4) yields $q_{\mathrm{m}}=-88.8 \pm 3.5 e$, which is nearly identical to the theoretically expected effective charge $q_{\mathrm{c}}=-89.6 e$ for the disordered protein Stm-1 [19,23]. Stm-1 thus serves as the "calibration molecule," which determines the value of the surface potential in Eq. (4) that should hold for all measurements under the same 


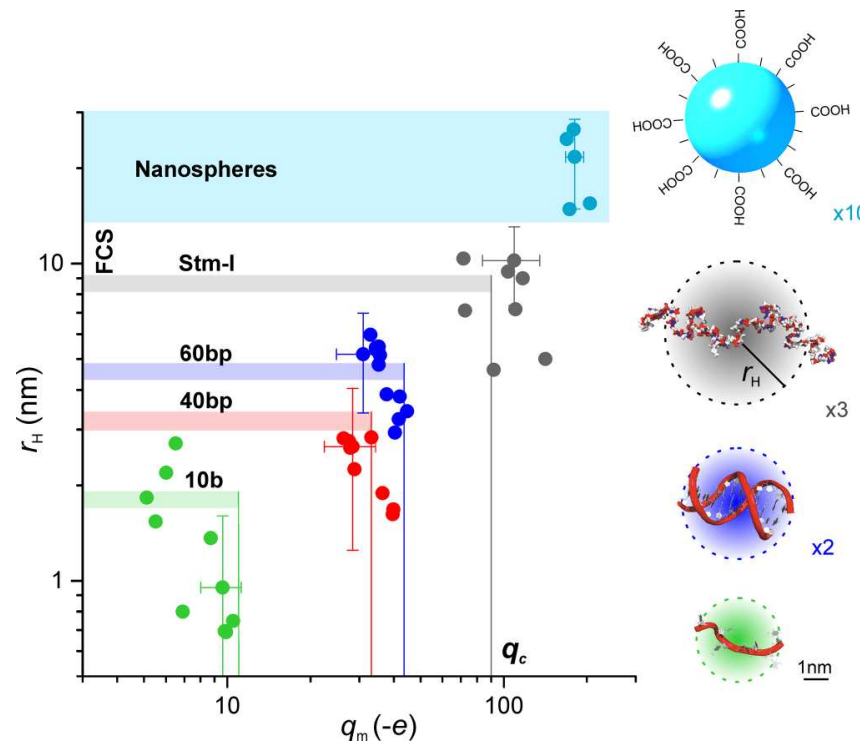

FIG. 7. Measurements of hydrodynamic radius and effective electrical charge of single biomolecules in solution. Each data symbol corresponds to the information obtained for a single object, from top to bottom: 24-nm-radius FluoSpheres (light blue), an intrinsically disordered protein, Stm-l (gray), 60 bp dsDNA (blue), 40 bp dsDNA (red), and 10 base ssDNA (green). Error bars are standard error of the mean (s.e.m.) and are presented for one molecule of each species. Vertical lines denote the theoretically expected charge, $q_{\mathrm{c}}$, while horizontal bands correspond to radius measurements from fluorescence correlation spectroscopy (FCS) on molecules and DLS for particles (band thickness indicates measurement standard deviation). On the right, structural representations of the measured species are reduced by the scaling factor noted.

conditions. Note that an effective surface potential of $2.8 k_{\mathrm{B}} T / e$ corresponds to a value of $\mathrm{p} K_{\mathrm{a}}=9.5$ in a charge regulation model of the silica-water interface surface, excluding the Stern layer $[23,40]$. This value is consistent with trends emerging from the most recent experimental and theoretical work on amorphous silica surfaces [41,42].

We introduce solutions of each molecular species into the free-energy landscape and record typically 10 molecular migration trajectories in each case. We report measurements on 10 base single-stranded (ss) DNA, 40 bp double-stranded (ds) DNA, $60 \mathrm{bp}$ dsDNA, and and the starmaker-like intrinsically disordered protein (Stm-l, molecular weight $40 \mathrm{kDa}$ ) [20]. The effective charge and hydrodynamic radius values for these molecular species span a range of about one order of magnitude. The combination of measured $t_{\mathrm{ON}}$ and $t_{\mathrm{OFF}}$ values for each molecule together determines its measured effective charge $q_{\mathrm{m}}$ as well as its hydrodynamic radius, $r_{\mathrm{H}}$, as shown in Fig. 7. $q_{\mathrm{m}}$ and $r_{\mathrm{H}}$ values averaged over about 10 molecules for each species are shown in Table I.

Note that since the data on each molecular species were not averaged over several independent experiments, the accuracy on all the reported charge values in this work is not expected to be better than within $\sim 20 \%$ due to the ca. $1 \mathrm{~nm}$ uncertainty in determining the slit height, $2 h$, in a single experiment. The accuracy at the level of the single measurement can be improved using sequential measurement in the same lattice that

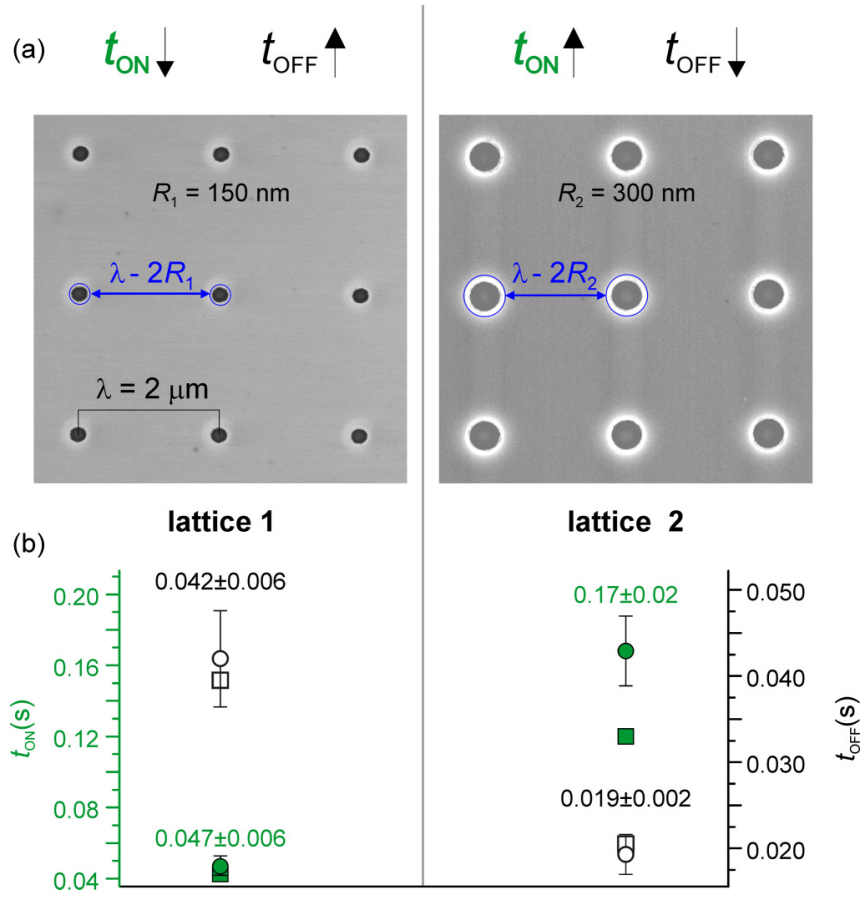

FIG. 8. (a) SEM images of two lattices of pitch $\lambda=2 \mu \mathrm{m}$ and nanostructure radius, $R_{1}=150 \mathrm{~nm}$ (left) and $R_{2}=300 \mathrm{~nm}$ (right). Solid circles represent the circumferences of the surface nanostructures and the effective lattice pitch, $\lambda_{i}^{\prime}=\lambda-2 R_{i}$, is indicated in blue. (b) The plot compares the experimentally measured time scales (circles) for the Stm-1 molecule with those expected from the simulation (squares), where $q_{\mathrm{eff}}=q_{\mathrm{c}}=-89.6 e$ and $r_{\mathrm{H}}=r_{\mathrm{H}, \mathrm{FCS}}=$ $8.7 \mathrm{~nm}$. Filled symbols represent $t_{\mathrm{ON}}$ and open symbols give $t_{\mathrm{OFF}}$ values. Reported experimental values are averages over data from five molecules. The errors on the simulated values are smaller than the symbols.

entails first measuring a known standard and then the molecule of interest, or by parallel measurement on spectrally resolvable molecules [19]. Doing so would permit highly accurate single measurements with statistically limited precision.

We further subjected the measurement concept to a test of robustness with respect to the choice of landscape parameters, namely effective lattice pitch and radius of the potential wells. We report measurements performed on the Stm-l molecule in lattices with different nanostructure radii, $R_{1}=150 \mathrm{~nm}$ and $R_{2}=300 \mathrm{~nm}$, but identical pitch $\lambda=2 \mu \mathrm{m}$ [Fig. 8(a)]. We expect both time scales to respond to this change in lattice geometry: on-times, $t_{\mathrm{ON}}$, depend on the area of the trapping nanostructures, while off-times, $t_{\mathrm{OFF}}$, are expected to scale with the area of the "field-free" region of the lattice. In fact, we find that $t_{\mathrm{ON}}$ values measured in lattice 2 are 3.6 times larger than in lattice 1 , close to the expected value, $\left(R_{2} / R_{1}\right)^{2}=4$. Conversely, in lattice 2 , the measured $t_{\mathrm{OFF}}$ is smaller than the value in lattice 1 by a factor 2 . Here, the ratio of off-times is expected to correspond to the squared ratio of the effective lattice pitch values, $\left(\lambda_{1}^{\prime} / \lambda_{2}^{\prime}\right)^{2}=1.8 \pm 0.1$, where $\lambda_{i}^{\prime} \approx \lambda-2 R_{i}$. The measured data are in remarkable agreement with the simulation predictions for a molecule of effective charge, $q_{\mathrm{eff}}=q_{\mathrm{c}}=-89.6 e$ and $r_{\mathrm{H}}=r_{\mathrm{H}, \mathrm{FCS}}=8.7 \mathrm{~nm}$, which correspond to the properties of Stm-1 [Fig. 8(b)]. 

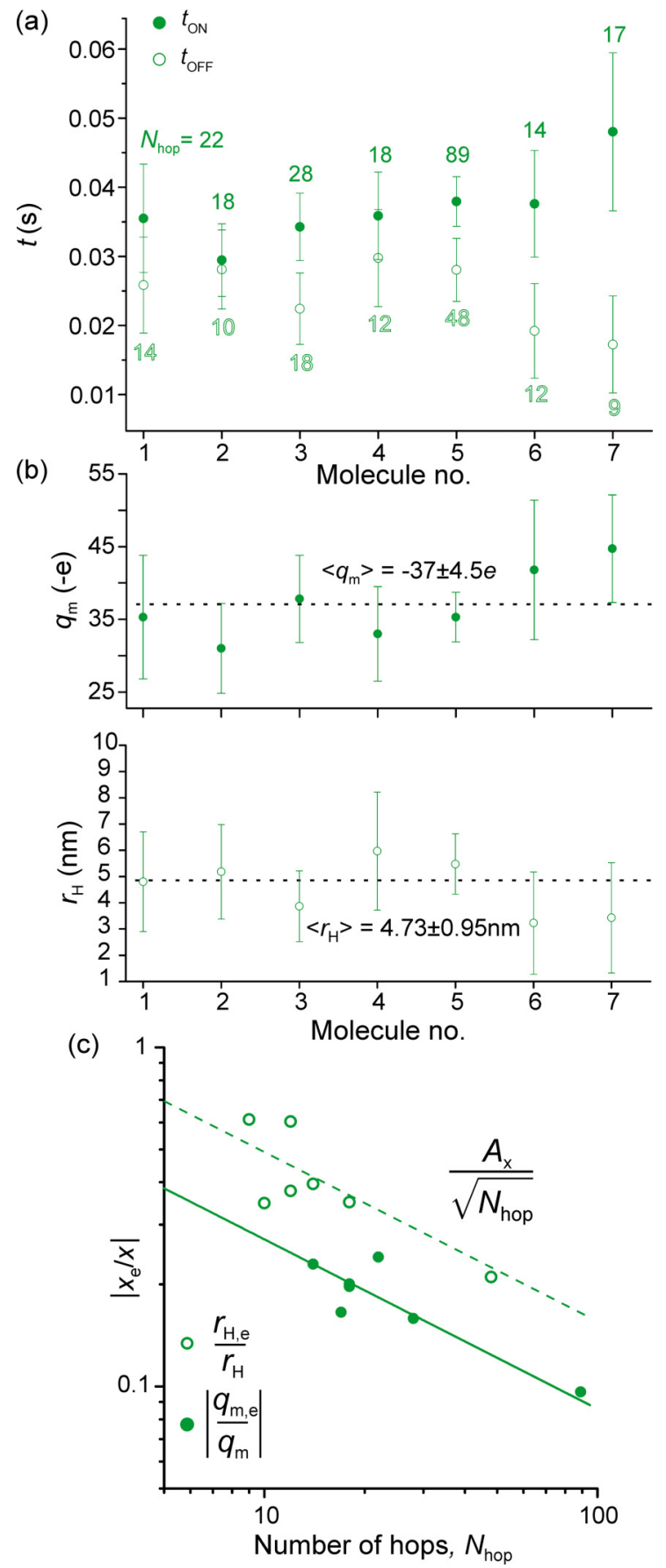

FIG. 9. (a) Measurements of $t_{\mathrm{OFF}}$ (empty symbols) and $t_{\mathrm{ON}}$ (solid green symbols) for representative single $60 \mathrm{bp}$ dsDNA molecules. Error bars denote s.e.m. over the number of hops, $N_{\text {hop }}$, indicated by each symbol. (b) Inferred $r_{\mathrm{H}}$ and $q_{\mathrm{m}}$ values for each molecule in panel (a). (c) Plots of measured fractional errors on $r_{\mathrm{H}}$ and $q_{\mathrm{m}} \mathrm{vs}$ $N_{\text {hop }}$ for the data in panel (a). The lines represent fits of the form $\left|x_{e} / x\right|=A_{\mathrm{x}} / \sqrt{N_{\mathrm{hop}}}$, where $x$ denotes the measurable: either $q_{\mathrm{m}}$ or $r_{\mathrm{H}}$. For $r_{\mathrm{H}}$, we find $A_{r}=1.7 \pm 0.1$, in good agreement with the expected value of $1.9 \pm 0.15$, while for $q_{\mathrm{m}}$ we obtain $A_{q}=0.87 \pm 0.05$, close to the expected value of 0.82 (see Appendix D for error propagation).

Next we focus on the experimentally attained precision in our single molecule measurements. Data acquired on representative single molecules of $60 \mathrm{bp}$ dsDNA with the values of $N_{\text {hop }}$ ranging from 5 to 100 are shown in Fig. 9. (a)

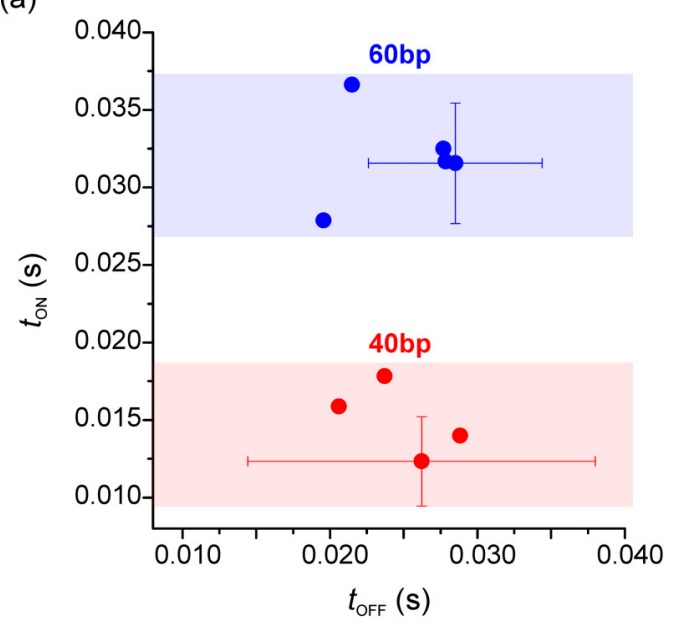

(b)

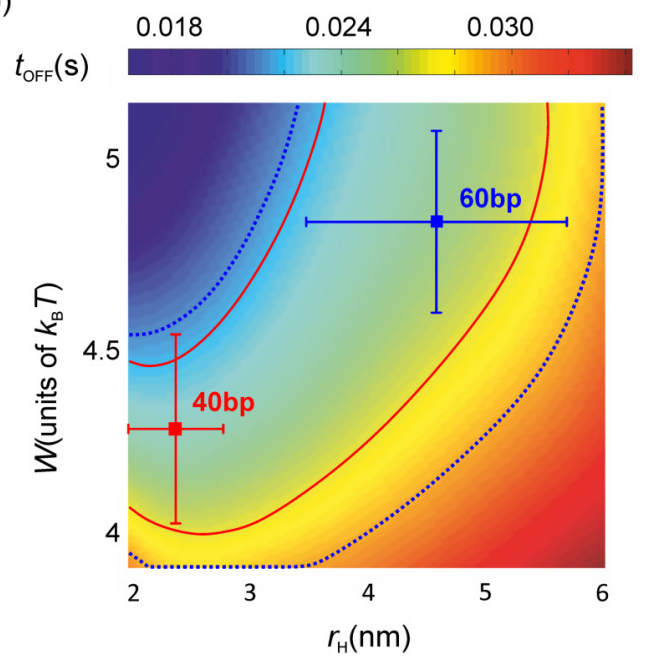

(c)

\begin{tabular}{lcccc} 
& $\begin{array}{c}\boldsymbol{r}_{\mathrm{H}} \\
(\mathrm{nm})\end{array}$ & $\begin{array}{c}\boldsymbol{r}_{\mathrm{H}, \mathrm{Fcs}} \\
(\mathbf{n m})\end{array}$ & $\begin{array}{c}\boldsymbol{q}_{\mathbf{m}} \\
(-\mathrm{e})\end{array}$ & $\begin{array}{c}\boldsymbol{q}_{\mathrm{c}} \\
(-\mathrm{e})\end{array}$ \\
\cline { 2 - 5 } 40bp & $2.4 \pm 0.4$ & $3.2 \pm 0.2$ & $32 \pm 3.6$ & 32.7 \\
60bp & $4.6 \pm 1.1$ & $4.5 \pm 0.2$ & $38.4 \pm 4.2$ & 43.4 \\
\hline
\end{tabular}

FIG. 10. (a) Measurements of $t_{\mathrm{OFF}}$ and $t_{\mathrm{ON}}$ compared for $\sim 5$ molecules of $40 \mathrm{bp}$ (red symbols) and $60 \mathrm{bp}$ dsDNA (blue symbols). While the values of $t_{\mathrm{OFF}}$ are nearly indistinguishable $\left(\left\langle t_{\mathrm{OFF}, 40 \mathrm{bp}}\right\rangle=\right.$ $24.4 \pm 3 \mathrm{~ms}$ and $\left.\left\langle t_{\mathrm{OFF}, 60 \mathrm{bp}}\right\rangle=25 \pm 4 \mathrm{~ms}\right)$, the simultaneously measured on-times, $t_{\mathrm{ON}}$, are well resolved $\left(\left\langle t_{\mathrm{ON}, 40 \mathrm{bp}}\right\rangle=15 \pm 2 \mathrm{~ms}\right.$ and $\left.\left\langle t_{\mathrm{ON}, 60 \mathrm{bp}}\right\rangle=32 \pm 3 \mathrm{~ms}\right)$. (b) A surface plot of $t_{\mathrm{OFF}}=f\left(W, r_{\mathrm{H}}\right)$ reveals nonmonotonic behavior of $t_{\mathrm{OFF}}$ in the low $\left(W, r_{\mathrm{H}}\right)$ regime which results in similar $\left\langle t_{\mathrm{OFF}}\right\rangle$ values for the two species, despite their different hydrodynamic radii. Overlapping isobands of measured $\left\langle t_{\mathrm{OFF}}\right\rangle$ for 40 and $60 \mathrm{bp}$ dsDNA molecules are demarcated by dotted (60 bp) and solid (40 bp) contour lines. Including information on $t_{\mathrm{ON}}$, however, permits unique values of $W$ and $r_{\mathrm{H}}$ to be assigned to each species (square symbols). (c) Tabulated averages of measured $q_{\mathrm{m}}$ and $r_{\mathrm{H}}$ reveal good agreement with the theoretically expected values of $q_{\mathrm{c}}$ and $r_{\mathrm{H}}$, independently measured via FCS.

Figure 9(c) shows that the precision we obtain on measurements of both $q_{\mathrm{m}}$ and $r_{\mathrm{H}}$ is statistically limited, with $N_{\text {hop }}$ currently determined by the field of view in the imaging system. Clearly, improvements on this front will lead to more 
precise measurements on a single molecule, approaching at least the precision shown in the measurements in Table I.

Finally, we demonstrate an interesting effect in the experiment that can arise in the measurement of two similar molecular species. We observe that the comparatively weak trapping of a smaller, less charged molecule-say, species 1 (40 bp DNA) — can result in a measured $t_{\mathrm{OFF}}$ value comparable in magnitude to that obtained for the larger, more highly charged, and more stably trapped molecule of species 2 (60 bp DNA) [Fig. 10(a)]. An intuitive conclusion from the magnitudes of the measured ( $t_{\mathrm{ON}}, t_{\mathrm{OFF}}$ ) data would point to different effective charges but the same hydrodynamic radius for both species. Notably, however, the simulation analysis assigns $\left(q_{\mathrm{m}}, r_{\mathrm{H}}\right)$ values to the measured $\left(t_{\mathrm{ON}}, t_{\mathrm{OFF}}\right)$ data that are in very good agreement with expectations for both molecules, where not only the effective charge values but also the hydrodynamic radii are significantly different [Fig. 10(c)].

The reason behind this counterintuitive observation is due to a well-depth-dependent effect on the dynamics. Free-diffusive transport terminates once a molecule is absorbed or trapped at a lattice site. If the field at the periphery of the potential well is strong, which generally implies that $W$ is large, the capture probability is high, which means that a molecule that samples this region will very likely be rapidly drawn to the bottom of the well and free diffusive transport terminates. For low values of $W$, however, the force toward the bottom of the well is smaller, and on short time scales the molecule is statistically highly likely to return to the free diffusion zone; this inflates the measured $t_{\mathrm{OFF}}$ in an experiment (Fig. 13 in Appendix C). In general, we find that return probabilities broadly defined, within the finite observation window, strongly influence measured time scales. Correctly accounting for the time-averaged dynamics and well-depth-dependent effects is therefore critical for accurate conversion of the measured time scales into physical parameters of interest.

\section{CONCLUSIONS}

Our study introduces lattice diffusion in an electrostatic landscape as a new experimental route to simultaneously measure both the hydrodynamic radius and the effective electrical charge of a single molecule in solution. The approach relies on standard wide-field optical microscopic observation of single fluorescently labeled molecules and also lends itself well to label-free imaging and measurement techniques $[43,44]$. Although our initial effort to establish the concept required an in-depth simulation-based analysis of Brownian motion in a 2D landscape, we point out that such studies yield simple functional dependences of the expected time scales on the magnitudes of the relevant physical properties of interest [Figs. 5(c) and 5(d)]. These relationships can then be broadly and directly applied to future measurements with simple rescaling to account for variations in experimental or system parameters.

Our combination of experiment and simulation in a periodic landscape has further revealed interesting features relevant for interpreting and understanding experimental observations on diffusive transport in a periodic potential. For example, while for large values of $W, t_{\mathrm{OFF}}$ decreases monotonically with decreasing $r_{\mathrm{H}}$ and is independent of well depth, as intuitively expected, we find interesting nonmonotonic behavior for small $W\left(<5 k_{\mathrm{B}} T\right)$. Here the measured time spent by an object of a given $r_{\mathrm{H}}$ in the field-free region of the lattice can be larger than that expected for high $W$ [Figs. 5(d) and 10(b)]. We find that this effect is due to the finite return probability of the molecule spatially sampling the edge of the well (Appendix C). In other words, the behavior in the regimes of high and low $W$ (or equivalently, effective charge, $q_{\text {eff }}$ ) are qualitatively delineated by the gradient of the potential at the periphery of the trap.

Further, the experiment we report holds significance for single-molecule studies, both in terms of direct optical measurement of static and dynamic molecular structural properties as well as in order to understand and interpret experimental observations of molecular transport in natural periodic systems. Since both the hydrodynamic radius and effective charge of a macromolecule depend on molecular 3D conformation, our new measurement principle paves the way toward experiments that explore static 3D conformation, or slow temporal changes of conformation, in a single macromolecule in solution.

\section{ACKNOWLEDGMENTS}

We gratefully acknowledge the Schweizerischer Nationalfonds zur Förderung der Wissenschaftlichen Forschung (SNSF), the European Research Council, and University of Zürich for financial support. We thank Ben Schuler and Andrzej Ożyhar for the gift of the Stm-l protein. Nanofabrication was carried out at FIRST Center for Micro- and Nanoscience, ETH Zürich.

\section{APPENDIX A: DEPENDENCE OF ON-TIME ON THE LOCATION OF THE ESCAPE BOUNDARY}

We analyzed the dependence of $t_{\mathrm{ON}}$ on the location of the the escape boundary, $r_{\mathrm{esc}}$ using BD simulations. For a lattice of pitch $\lambda=2 \mu \mathrm{m}$, we examine molecular residence times in two different regimes: $l_{\text {diff }} \gtrsim \lambda$, corresponding to a molecule of $r_{\mathrm{H}}=1 \mathrm{~nm}$, and $l_{\text {diff }}<\lambda$ for a molecule of $r_{\mathrm{H}}=20 \mathrm{~nm}$. In the former regime, we find that $t_{\mathrm{ON}}$ is nearly insensitive to $r_{\mathrm{esc}}$ while in the latter we note up to a factor 2 variation in $t_{\mathrm{ON}}$ depending on $r_{\text {esc }}$ [Fig. 11(b)].

\section{APPENDIX B: DETECTED OPTICAL SIGNAL FROM MOLECULAR MIGRATION IN THE LATTICE}

A lattice area of $100 \mu \mathrm{m}^{2}$, typically containing $\sim 20$ lattice sites, is monitored via wide-field fluorescence microscopy. Figure 12(a) shows a subset of a typical array that can be sampled by a single molecule during 1-10 s of observation time. The mean fluorescence intensity of each trap is monitored separately in time. The traces are then rescaled, binarized, and superimposed on each other in order to obtain the overall temporal lattice intensity signal [bold black line in Fig. 12(b)]. The high-intensity bursts in the trace give the time spent at a lattice site and the average thereof yields $t_{\mathrm{ON}}$. The average "dark time" in the trace in which none of the lattice sites appears occupied gives $t_{\mathrm{OFF}}$. 
(a)
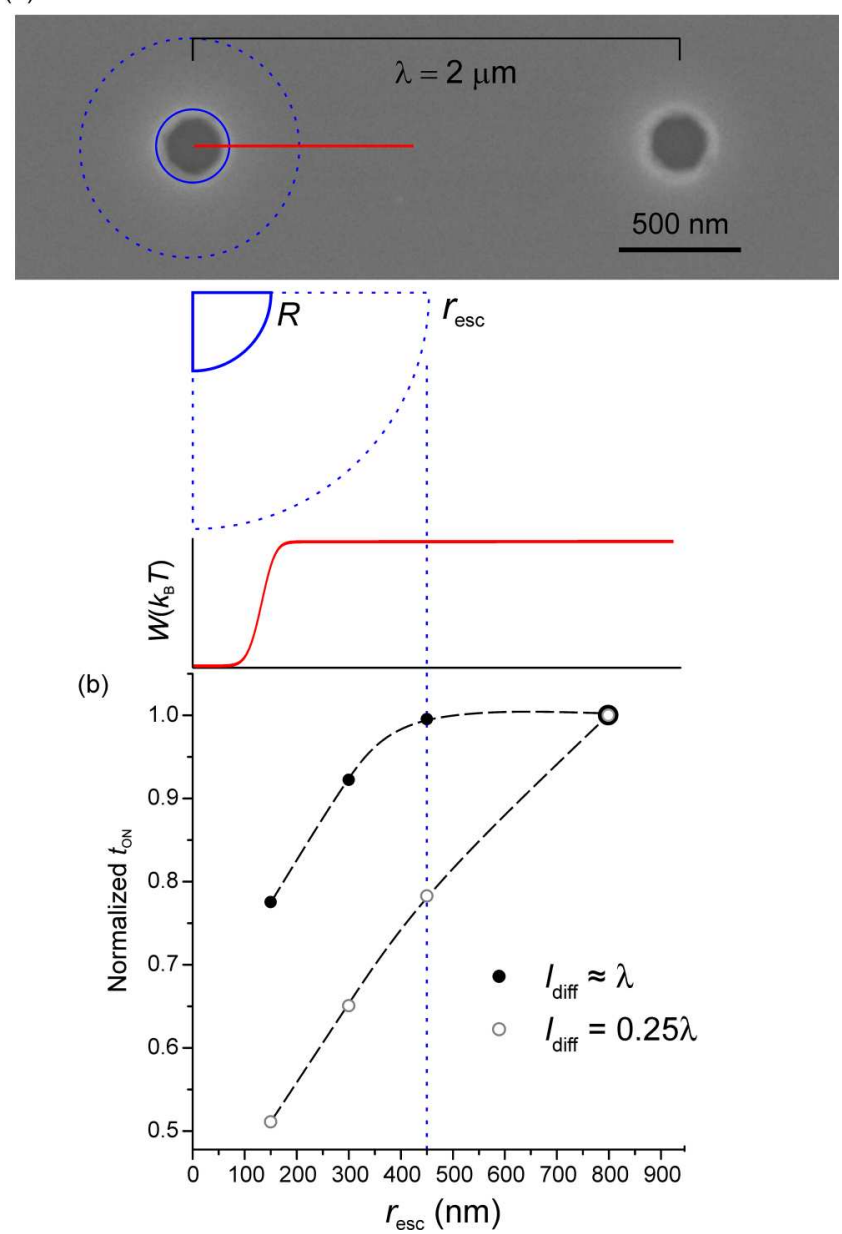

FIG. 11. (a) An SEM image of two trapping nanostructures of radius, $R=150 \mathrm{~nm}$ at a pitch $\lambda=2 \mu \mathrm{m}$. The solid circle denotes the circumference of the nanostructure, while the broken circle of radius $r_{\text {esc }}$ indicates the escape boundary. The red solid line in the lower panel represents the radial electrostatic free energy profile along the red line in the image above. (b) The simulated value of $t_{\mathrm{ON}}$ obtained for each $r_{\text {esc }}$ is normalized by the value obtained for $r_{\text {esc }}=800 \mathrm{~nm}$. The simulated dataset for $r_{\mathrm{H}}=1 \mathrm{~nm}\left(\lambda \approx l_{\text {diff }}\right)$ shows a weak dependance on $r_{\text {esc }}$ (black symbols). For $r_{\mathrm{H}}=20 \mathrm{~nm}$, we find a strong variation in this time scale, as the average molecular diffusion length is smaller than the lattice pitch (gray open symbols). The vertical dotted line indicates the $r_{\text {esc }}$ used for the analysis of the single-molecule transport for the depicted lattice geometry.

\section{APPENDIX C: DEPENDENCE OF OFF-TIME ON WELL DEPTH}

In order to study the dependance of $t_{\mathrm{OFF}}$ on well depth $W$ and in particular its nonlinear behavior for $W<5 k_{\mathrm{B}} T$, we performed BD simulations. We studied the probability of capture of a molecule at traps in the lattice. The capture probability is defined as the ratio of the number of trapped events, $n\left(\Delta t_{\mathrm{ON}}\right)$ to the total number of events capable of resulting in capture. The latter quantity is given by the number of instances in the trajectory that satisfy $\langle r\rangle_{t_{\mathrm{exp}}}<r_{\mathrm{esc}}$, with reference to each trap in the lattice.

For a molecule of $r_{\mathrm{H}}=3 \mathrm{~nm}$, we find than the probability of being captured by a well decreases with decreasing well depth (a)

(b)
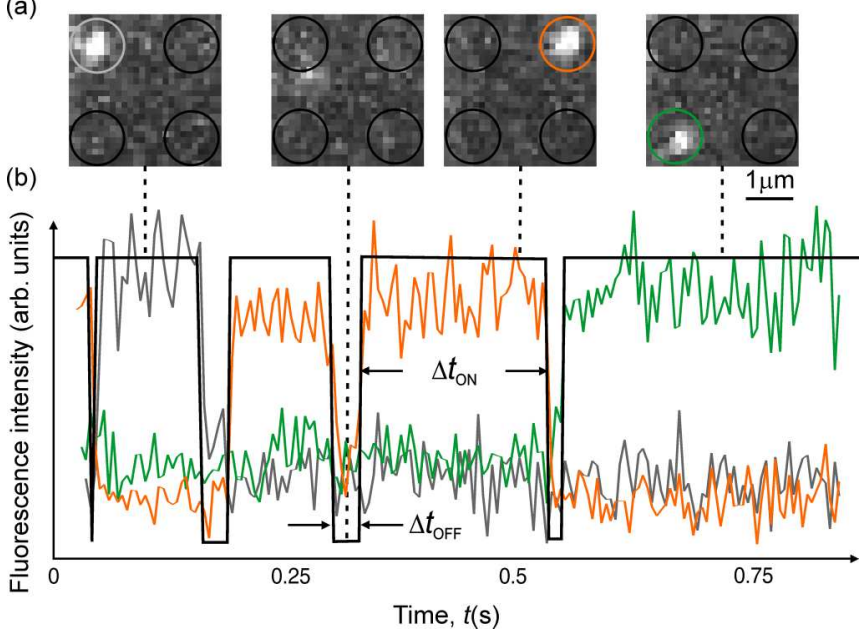

FIG. 12. (a) Wide-field fluorescence images of a lattice of four trapping nanostructures of $R=150 \mathrm{~nm}$ and $\lambda=2 \mu \mathrm{m}$. The average intensity of each circular region of interest (ROI) (black), centered on the lattice sites, is monitored in time. (b) Temporal intensity traces corresponding to three individual lattice sites are denoted by a different color. When the molecule occupies a lattice, the intensity recorded in the corresponding ROI is high, while the others appear dark. Each recorded trace is rescaled and binarized in order to compensate for heterogeneity in the illumination across the entire field of view.

[Fig. 13(b)]. This is due to the fact that when the trapping force is weak, a small rapidly diffusing molecule sampling the edge of the potential well can evade escape and return to the "field-free" zone, all within a time period $<t_{\exp }$. This gives rise to a longer lived $t_{\mathrm{OFF}}$ state. By contrast, at greater well depths a molecule sampling the edge of the well is very likely to reach the bottom and remain trapped for at least $t=t_{\exp }$, as reflected in the increased capture probability. In general, for larger molecules [e.g., $r_{\mathrm{H}}=9 \mathrm{~nm}$, Fig. 13(b)], given the same exposure time in the observation, the influence of well depth on the capture probability is weaker, as the diffusive dynamics is slower.

\section{APPENDIX D: ESTIMATING MEASUREMENT UNCERTAINTIES}

The measurement error, $x_{\mathrm{e}}$ on a quantity $x$, which is a function of the variables $f, g, h$, etc., each with uncertainties $f_{\mathrm{e}}, g_{\mathrm{e}}, h_{\mathrm{e}}$, etc., can be expressed as follows:

$$
x_{\mathrm{e}}=\sqrt{\left(\frac{\partial x}{\partial f}\right)^{2} f_{\mathrm{e}}^{2}+\left(\frac{\partial x}{\partial g}\right)^{2} g_{\mathrm{e}}^{2}+\left(\frac{\partial x}{\partial h}\right)^{2} h_{\mathrm{e}}^{2}+\cdots}
$$

In our experiment, the main source of error in the determination of both molecular effective charge, $q_{\mathrm{eff}}$, and hydrodynamic radius, $r_{\mathrm{H}}$, arises from the statistical uncertainty in measuring $t_{\mathrm{ON}}$ and $t_{\mathrm{OFF}}$ in a temporally limited experiment. Here both time scales, $\Delta t_{\mathrm{x}}$ (where $x$ is either ON or OFF) are exponentially distributed. Thus, the measurement error $t_{\mathrm{x}, \mathrm{e}}$ on their average value, $t_{\mathrm{x}}$, is function of the number of detected events or hops, 
(a) No capture Capture

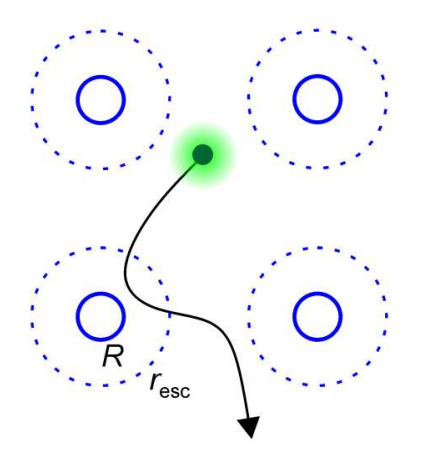

(b)

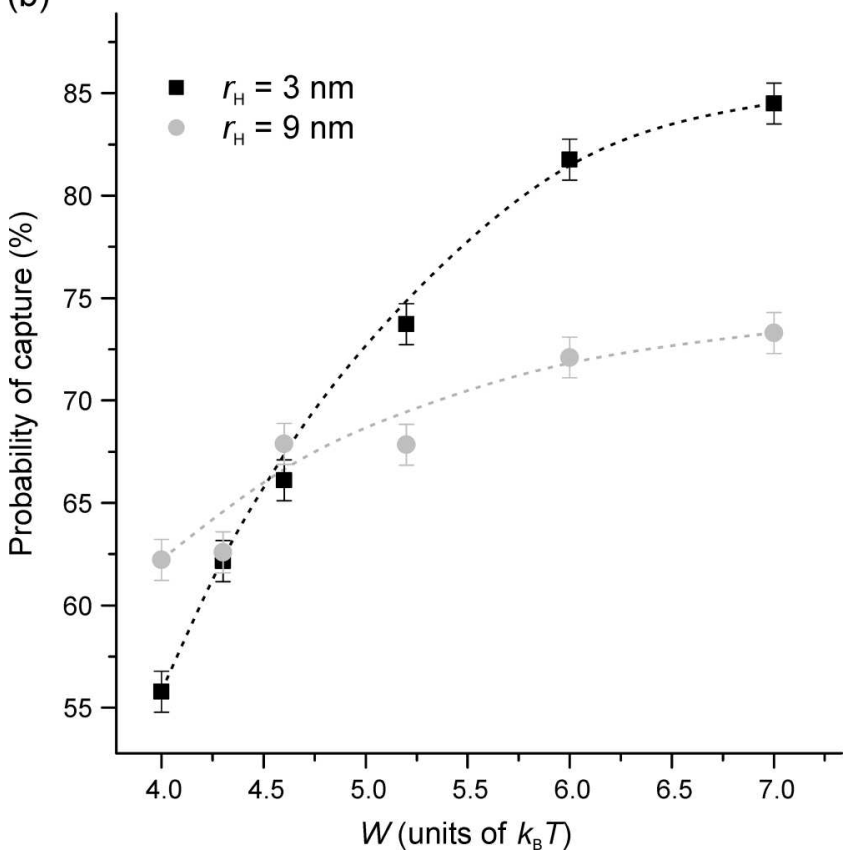

FIG. 13. (a) Schematic of a particle sampling a lattice of four traps. The blue solid line indicates the circumference of the surface indentations of radius $R$, while the dotted blue line shows the escape boundary $r_{\text {esc }}$. The molecule is considered "captured" if its trajectory results in a trapped state for a period $\geqslant t_{\exp }$ once it traverses $r_{\text {esc }}$. (b) The probability of a molecule being trapped once it samples the area within the escape boundary of a given pocket increases with increasing well depth for a molecule of $r_{\mathrm{H}}=3 \mathrm{~nm}$ (black squares), while the response is less pronounced for a molecule of larger radius $\left(r_{\mathrm{H}}=9 \mathrm{~nm}\right.$, gray circles). Dashed lines are guides to the eye.

$N_{\text {hop }}$, as follows:

$$
t_{\mathrm{x}, \mathrm{e}}=\frac{t_{\mathrm{x}}}{\sqrt{N_{\mathrm{hop}}}} .
$$

First, we consider the measurement error on $r_{\mathrm{H}}$. The measurement uncertainty on $r_{\mathrm{H}}$ is nearly entirely due to the statistical error in measuring an average off-time from a limited number of hops. We have established that the relationship between $t_{\mathrm{OFF}}$ and the diffusion coefficient of the molecule of interest, $D$, is well described by $t_{\mathrm{OFF}}=\lambda^{\prime 2} / 4 D$, where $\lambda^{\prime}$ is the effective lattice pitch. This expression can be rewritten in terms of $r_{\mathrm{H}}$ using the Stokes-Einstein relation as follows:

$$
r_{\mathrm{H}}=\frac{4 k_{\mathrm{B}} T}{6 \pi \eta \lambda^{\prime 2}} t_{\mathrm{OFF}}=a_{1} t_{\mathrm{OFF}} .
$$

Equation (D1) then gives the uncertainty on the hydrodynamic radius, $r_{\mathrm{H}, \mathrm{e}}$ :

$$
r_{\mathrm{H}, \mathrm{e}}=\sqrt{\left(\frac{\partial r_{\mathrm{H}}}{\partial t_{\mathrm{OFF}}}\right)^{2} t_{\mathrm{OFF}, \mathrm{e}}^{2}}=a_{1} t_{\mathrm{OFF}, \mathrm{e}} .
$$

Using the expression for $t_{\mathrm{OFF}, \mathrm{e}}$ given by Eq. (D2), we have for the fractional uncertainty on $r_{\mathrm{H}}$

$$
\frac{r_{\mathrm{H}, \mathrm{e}}}{r_{\mathrm{H}}}=\frac{a_{1}}{r_{\mathrm{H}}} \frac{t_{\mathrm{OFF}}}{\sqrt{N_{\mathrm{hop}}}}=\frac{A_{\mathrm{r}}}{\sqrt{N_{\mathrm{hop}}}} .
$$

Holding other parameters constant, the measurement error may be decreased by tuning the parameter $a_{1}$, which is inversely proportional to the squared effective pitch $\lambda^{\prime}$. Clearly, a large lattice pitch would improve the accuracy of the method; however, the lattice pitch is limited by the field of view in the measurement. On the other hand, for very small $\lambda^{\prime}$, the molecule would see a landscape of contiguous potential wells and never appear in the off-state, thus hindering the measurement. For the conditions of the experiment illustrated in Fig. 9, we determine the value of $A_{\mathrm{r}}=a_{1} t_{\mathrm{OFF}} / r_{\mathrm{H}}$ to be $1.9 \pm 0.15\left(\lambda^{\prime}=1.6 \pm 0.05 \mu \mathrm{m}, r_{\mathrm{H}}=4.7 \mathrm{~nm}\right.$, and $t_{\mathrm{OFF}}=$ $25.5 \mathrm{~ms})$. This is in good agreement with the experimental fit value $1.7 \pm 0.1$ in Fig. 9(c). Thus, $10 \%$ measurement error on $t_{\mathrm{OFF}}\left(N_{\mathrm{hop}}=100\right)$ would correspond to $\sim 17 \%$ error on $r_{\mathrm{H}}$.

Next, we estimate the error on the measured effective charge, $q_{\mathrm{m}}$, of the molecule. As described in the Supporting Information of our previous work [19], $q_{\text {eff }}$, hereafter referred to as $q$, depends on the measured time scale $t_{\mathrm{ON}}$ as follows:

$$
q \psi_{\mathrm{m}}+f=k_{\mathrm{B}} T \ln \left(\frac{t_{\mathrm{ON}}}{t_{\mathrm{r}}}\right) .
$$

Here $\psi_{\mathrm{m}}$ denotes the electrical potential at the midplane of the slit and $f$ is a particle fluctuation contribution to the total trap depth, $W$. This contribution is almost entirely entropic in origin and is essentially independent of $q . t_{\mathrm{r}}$ in turn reflects a position relaxation time of the particle, which again can be written in terms of a diffusion time scale, $t_{\mathrm{r}}=L^{2} / 4 D$. Here $L$ is a length scale on the order of $r_{\text {esc }}$, the radius of the escape boundary. The position relaxation time can therefore be rewritten in terms of $r_{\mathrm{H}}$ as $t_{\mathrm{r}}=\frac{L^{2}}{4 k_{\mathrm{B}} T} 6 \pi \eta r_{\mathrm{H}}=a_{2} r_{\mathrm{H}}$.

Equation (D6) now reads

$$
q \psi_{\mathrm{m}}+f=k_{\mathrm{B}} T \ln \left(\frac{t_{\mathrm{ON}}}{a_{2} r_{\mathrm{H}}}\right) .
$$

Thus the fractional error $q_{\mathrm{e}} / q$ according to Eq. (D1) is

$$
\begin{aligned}
\frac{q_{\mathrm{e}}}{q}= & \frac{\sqrt{\left(\frac{1}{q^{2}}\right)\left(\frac{\partial q}{\partial \psi_{\mathrm{m}}}\right)^{2} \psi_{\mathrm{m}, \mathrm{e}}^{2}+\left(\frac{1}{q^{2}}\right)\left(\frac{\partial q}{\partial t_{\mathrm{ON}}}\right)^{2} t_{\mathrm{ON}, \mathrm{e}}^{2}}}{+\left(\frac{1}{q^{2}}\right)\left(\frac{\partial q}{\partial r_{\mathrm{H}}}\right)^{2} r_{\mathrm{H}, \mathrm{e}}^{2}}
\end{aligned}
$$

The first term in Eq. (D8) represents the uncertainty in the determination of the midplane potential. This aspect has been discussed at length in our previous work [19], and arises 
from the uncertainty in determining the slit height with an accuracy better than $h_{\mathrm{e}}=1 \mathrm{~nm}$. For a molecule whose radius is known a priori from an independent measurement, and where the escape dynamics is well sampled $\left(N_{\text {hop }}>100\right)$, as in our previous work, this first term would be the main source of error, and is estimated at about $6 \%$ when averaging over four to five independent experiments. In a single experiment, however, where the height of the slit maybe depart from the mean value by as much as 2 or $3 h_{\mathrm{e}}$, this (single) measurement inaccuracy can be as large as $18 \%$.

In the current experiment, short single-molecule trajectories are also affected by the statistical noise on $t_{\mathrm{ON}}$ (second term). It turns out that the total measurement error is in fact dominated by the measurement error on $r_{\mathrm{H}}$ (third term), which is measured simultaneously here.

As reflected in Eq. (D5), $r_{\mathrm{H}, \mathrm{e}}$ itself is a function of the number of hops, $N_{\text {hop. }}$. The second and third terms thus essentially represent the error due to statistical noise, $\left(q / q_{\mathrm{e}}\right)_{\mathrm{N}}$, and can be written as follows:

$$
\begin{aligned}
& \left(\frac{q \mathrm{e}}{q}\right)_{\mathrm{N}} \\
& =\sqrt{\left(\frac{1}{q^{2}}\right)\left(\frac{k_{\mathrm{B}} T}{t_{\mathrm{ON}} \psi_{\mathrm{m}}}\right)^{2} t_{\mathrm{ON}, \mathrm{e}}^{2}+\left(\frac{1}{q^{2}}\right)\left(-\frac{k_{\mathrm{B}} T}{\psi_{\mathrm{m}} r_{\mathrm{H}}}\right)^{2} r_{\mathrm{H}, \mathrm{e}}^{2}}
\end{aligned}
$$

(D9) which simplifies to

$$
\left(\frac{q \mathrm{e}}{q}\right)_{\mathrm{N}}=\frac{1}{\sqrt{N_{\mathrm{hop}}}} \frac{k_{\mathrm{B}} T}{q \psi_{m}} \sqrt{1+\left(\frac{a_{1} t_{\mathrm{OFF}}}{r_{\mathrm{H}}}\right)^{2}}=\frac{A_{\mathrm{q}}}{\sqrt{N_{\mathrm{hop}}}} .
$$

The first term in Eq. (D9) represents the contribution of statistical noise on $t_{\mathrm{ON}}$ to the overall measurement error. As noted in previous work, the logarithmic dependence of $q$ on the escape time (on-time) means that poorly sampled dynamics will still give a relatively precise charge measurement. Also, as the electrostatic well depth $q \psi_{\mathrm{m}}$ is larger, the attenuation of the statistical noise, $1 / \sqrt{N_{\text {hop }}}$, is greater. In this experiment, we have $\left\langle q \psi_{\mathrm{m}}\right\rangle=2.6 k_{\mathrm{B}} T$. Thus $10 \%$ error in determining $t_{\mathrm{ON}}$ corresponds to only $\sim 4 \%$ error on $q$. However, the second term in Eq. (D9)—which arises from the uncertainty on $r_{\mathrm{H}}$-contributes predominantly to the overall error. Under the experimental conditions concerned, we calculate $A_{\mathrm{q}}=0.82$, which is very close to the fit value $0.87 \pm 0.05$ from Fig. 9(c).

To conclude, given $N_{\text {hop }}=100$, we expect $\sim 9 \%$ error on the measured $q$ value arising from statistical uncertainty alone. Including the contribution from the uncertainty on the midplane potential, $\psi_{\mathrm{m}}$, we estimate an overall uncertainty or measurement inaccuracy of $\sim 20 \%$ in a single measurement.
[1] R. Gomer, Rep. Prog. Phys. 53, 917 (1990).

[2] G. Ayrault and G. Ehrlich, J. Chem. Phys. 60, 281 (1974).

[3] J. V. Barth, H. Brune, B. Fischer, J. Weckesser, and K. Kern, Phys. Rev. Lett. 84, 1732 (2000).

[4] B. G. Briner, M. Doering, H. P. Rust, and A. M. Bradshaw, Science 278, 257 (1997).

[5] H. X. Zhou, J. Phys. Chem. 94, 8794 (1990).

[6] P. Hanggi, P. Talkner, and M. Borkovec, Rev. Mod. Phys. 62, 251 (1990).

[7] J. Helenius, G. Brouhard, Y. Kalaidzidis, S. Diez, and J. Howard, Nature (London) 441, 115 (2006).

[8] J. Gorman, A. J. Plys, M.-L. Visnapuu, E. Alani, and E. C. Greene, Nat. Struct. Mol. Biol. 17, 932 (2010).

[9] X. G. Ma, P. Y. Lai, and P. G. Tong, Soft Matter 9, 8826 (2013).

[10] M. Pelton, K. Ladavac, and D. G. Grier, Phys. Rev. E 70, 031108 (2004).

[11] D. Kim, C. Bowman, J. T. Del Bonis-O’Donnell, A. Matzavinos, and D. Stein, Phys. Rev. Lett. 118, 048002 (2017).

[12] S. W. P. Turner, M. Cabodi, and H. G. Craighead, Phys. Rev. Lett. 88, 128103 (2002).

[13] V. Garcés-Chávez, R. Quidant, P. J. Reece, G. Badenes, L. Torner, and K. Dholakia, Phys. Rev. B 73, 085417 (2006).

[14] J. Fu, R. B. Schoch, A. L. Stevens, S. R. Tannenbaum, and J. Han, Nat. Nanotechnol. 2, 121 (2007).

[15] K. Xiao and D. G. Grier, Phys. Rev. Lett. 104, 028302 (2010).

[16] J. Skolnick, J. Chem. Phys. 145, 100901 (2016).

[17] J. A. Dix and A. S. Verkman, Annu. Rev. Biophys. 37, 247 (2008).
[18] M. Krishnan, N. Mojarad, P. Kukura, and V. Sandoghdar, Nature (London) 467, 692 (2010).

[19] F. Ruggeri, F. Zosel, N. Mutter, M. Różycka, M. Wojtas, A. Ożyhar, B. Schuler, and M. Krishnan, Nat. Nanotechnol. 12, 488 (2017).

[20] M. Różycka, M. Wojtas, M. Jakób, C. Stigloher, M. Grzeszkowiak, M. Mazur, and A. Ożyhar, PLoS ONE 9, e114308 (2014).

[21] H. Kramers, Phys. (Amsterdam, Neth.) 7, 284 (1940).

[22] M. Krishnan, J. Chem. Phys. 138, 114906 (2013).

[23] M. Krishnan, J. Chem. Phys. 146, 205101 (2017).

[24] N. Mojarad and M. Krishnan, Nat. Nanotechnol. 7, 448 (2012).

[25] See Supplemental Material at http://link.aps.org/supplemental/ 10.1103/PhysRevE.96.062406 for a movie of lattice diffusion of a single molecule.

[26] B. U. Felderhof, Phys. A (Amsterdam, Neth.) 387, 1767 (2008).

[27] B. U. Felderhof, Phys. A (Amsterdam, Neth.) 387, 39 (2008).

[28] S. Chandrasekhar, Rev. Mod. Phys. 15, 1 (1943).

[29] S. Lifson and J. L. Jackson, J. Chem. Phys. 36, 2410 (1962).

[30] D. L. Weaver, Phys. A (Amsterdam, Neth.) 98, 359 (1979).

[31] R. Zwanzig, Phys. A (Amsterdam, Neth.) 117, 277 (1983).

[32] M. Siler and P. Zemanek, in Conference on Optical Trapping and Optical Micromanipulation VII (SPIE, Bellingham, Washington, 2010), Vol. 7762.

[33] R. Verdel, L. Dagdug, A. M. Berezhkovskii, and S. M. Bezrukov, J. Chem. Phys. 144, 084106 (2016).

[34] Y. Su, P. Y. Lai, B. J. Ackerson, X. Cao, Y. Han, and P. Tong, J. Chem. Phys. 146, 214903 (2017).

[35] C. W. Gardiner, in Handbook of Stochastic Methods (SpringerVerlag, Berlin, Heidelberg, 1985), p. 140. 
[36] T. Savin and P. S. Doyle, Biophys. J. 88, 623 (2005).

[37] S. L. Eichmann, S. G. Anekal, and M. A. Bevan, Langmuir 24, 714 (2008).

[38] Y. Pawar and J. L. Anderson, Ind. Eng. Chem. Res. 32, 743 (1993).

[39] J. Happel and H. Brenner, in Low Reynolds Number Hydrodynamics (Springer, Netherlands, 1983), p. 286.

[40] S. H. Behrens and D. G. Grier, J. Chem. Phys. 115, 6716 (2001).
[41] M. Pfeiffer-Laplaud, D. Costa, F. Tielens, M.-P. Gaigeot, and M. Sulpizi, J. Phys. Chem. C 119, 27354 (2015).

[42] L. Dalstein, E. Potapova, and E. Tyrode, Phys. Chem. Chem. Phys. 19, 10343 (2017).

[43] D. Cole, G. Young, A. Weigel, A. Sebesta, and P. Kukura, ACS Photon. 4, 211 (2017).

[44] P. Kukura, M. Celebrano, A. Renn, and V. Sandoghdar, Nano Lett. 9, 926 (2009). 